

\title{
Counting rational points over number fields on a singular cubic surface
}

\author{
Christopher Frei
}

\begin{abstract}
A conjecture of Manin predicts the distribution of $K$-rational points on certain algebraic varieties defined over a number field $K$. In recent years, a method using universal torsors has been successfully applied to several hard special cases of Manin's conjecture over the field $\mathbb{Q}$. Combining this method with techniques developed by Schanuel, we give a proof of Manin's conjecture over arbitrary number fields for the singular cubic surface $S$ given by the equation $x_{0}^{3}=x_{1} x_{2} x_{3}$.
\end{abstract}

1. Introduction 1451

2. Passing to a universal torsor 1454

3. Proof of Theorem $1 \quad 1460$

4. Auxiliary results 1462

5. Proof of Lemma $3.1 \quad 1465$

$\begin{array}{ll}\text { References } & 1477\end{array}$

\section{Introduction}

We consider the cubic surface $S \subseteq \mathbb{P}^{3}$ defined over any number field $K$ by the equation

$$
x_{0}^{3}=x_{1} x_{2} x_{3} .
$$

It is toric, has three singular points $(0: 1: 0: 0),(0: 0: 1: 0),(0: 0: 0: 1)$, and contains three lines $L_{i}:=\left\{x_{0}=x_{i}=0\right\}$, for $i \in\{1,2,3\}$. The set $S(K)$ of $K$-rational points on $S$ is infinite.

The Weil height of $\boldsymbol{x}=\left(x_{0}: x_{1}: x_{2}: x_{3}\right) \in \mathbb{P}^{3}(K)$ is defined by

$$
H(\boldsymbol{x})=\prod_{\nu \in M(K)} \max \left\{\left|x_{0}\right|_{\nu},\left|x_{1}\right|_{\nu},\left|x_{2}\right|_{\nu},\left|x_{3}\right|_{\nu}\right\}^{d_{\nu}} .
$$

Here, $M(K)$ is the set of places of $K$, the absolute values $|\cdot|_{\nu}$ are normalized such that they extend the usual absolute values on $\mathbb{Q}$, and $d_{v}$ is the local degree $\left[K_{v}: \mathbb{Q}_{p}\right]$, if $v$ extends the place $p$ of $\mathbb{Q}$.

MSC2010: primary 11D45; secondary 14G05.

Keywords: Manin's conjecture, number fields, rational points, singular cubic surface. 
It is well known that there are only finitely many points of bounded height in $\mathbb{P}^{3}(K)$, so it makes sense to study the number of $K$-rational points on $S$ of height bounded by $B$, as $B$ tends to infinity. A generalization of a conjecture by Manin [Franke et al. 1989; Batyrev and Tschinkel 1998b], applied to our case, links the asymptotic behavior of this quantity to geometric features of $S$, provided that we exclude the points lying on the lines $L_{i}$. Indeed, the number of $K$-rational points of bounded height on these lines dominates the number of $K$-rational points on the rest of $S$, whereas much of the geometric information about $S$ would be lost when considering just the lines.

Therefore, we denote by $U$ the complement of the three lines in $S$ and define the counting function

$$
N(B):=|\{\boldsymbol{x} \in U(K) \mid H(\boldsymbol{x}) \leq B\}| .
$$

Here, $U(K)$ is the set of $K$-rational points on $U$. The above-mentioned generalization of Manin's conjecture [Franke et al. 1989; Batyrev and Tschinkel 1998b] to Fano varieties with at worst canonical singularities predicts in this case that

$$
N(B) \sim c B(\log B)^{6},
$$

with a positive leading constant $c=c_{S, K, H}$. A conjectural interpretation of the leading constant in Manin's conjecture was given by Peyre [1995] and extended to Fano varieties with at worst canonical singularities by Batyrev and Tschinkel [1998b]. When writing "Manin's conjecture", we implicitly include the conjecture about the leading constant.

Manin's conjecture has been proved for smooth toric varieties over arbitrary number fields by Batyrev and Tschinkel [1998a], studying the height zeta function with the help of Fourier analysis. In [Batyrev and Tschinkel 1998b] they explain how this result can be applied to prove Manin's conjecture for our singular surface $S$. Similar methods work for other varieties that are equivariant compactifications of certain algebraic groups; for example, see [Chambert-Loir and Tschinkel 2002].

Salberger [1998] gave a new proof of Manin's conjecture for split toric varieties over the field $\mathbb{Q}$ of rational numbers by a fundamentally different approach using universal torsors. These were first introduced by Colliot-Thélène and Sansuc [1980; 1987] to study the Hasse principle. In the context of Manin's conjecture, the basic idea is to find a parametrization of the rational points on the variety under consideration that makes it feasible to count them by analytic number theory.

Based on Salberger's ideas, proofs were found for several hard special cases of Manin's conjecture over $\mathbb{Q}$, to which the methods of Batyrev and Tschinkel cannot be applied; see for instance [Baier and Browning 2013; de la Bretèche 2002; de la Bretèche and Browning 2011; de la Bretèche et al. 2007; de la Bretèche and Fouvry 2004; de la Bretèche et al. 2012; Browning and Derenthal 2009; Le Boudec 2012]. 
For our surface $S$, independent proofs of Manin's conjecture over $\mathbb{Q}$ were given by de la Bretèche [1998], Fouvry [1998], Salberger [1998], Heath-Brown and Moroz [1999], and de la Bretèche and Swinnerton-Dyer [2007], with the help of such parametrizations. The best error terms have been obtained in [de la Bretèche 1998; de la Bretèche and Swinnerton-Dyer 2007].

In a first attempt to generalize universal torsor techniques to number fields other than $\mathbb{Q}$, Derenthal and Janda [2013] modified the approach by Heath-Brown and Moroz [1999] and successfully applied it to the case of imaginary quadratic number fields of class number 1.

In this article, we combine the method of Derenthal and Janda with ideas developed by Schanuel [1979] and apply it to arbitrary number fields. To the author's best knowledge, this is the first example of universal torsor techniques applied to a special case of Manin's conjecture over general number fields, aside from Schanuel's result for $\mathbb{P}^{n}$. Hopefully, similar approaches will lead to results for nontoric varieties.

Before we state the theorem, let us fix some notation: by $\Delta_{K}, h_{K}, R_{K}$, and $\omega_{K}$, we denote the discriminant, class number, regulator, and number of roots of unity of $K$. Moreover, $r$ and $s$ denote the number of real and complex places of $K$, and $q:=r+s-1$. We write $\mathbb{O}_{K}$ for the ring of integers of $K$ and $\mathfrak{N a}$ for the absolute norm of the nonzero fractional ideal $\mathfrak{a}$ of $K$.

Theorem 1. For every number field $K$, we have

$$
N(B)=c_{K} B(\log B)^{6}+O\left(B(\log B)^{5}\right),
$$

for $B \geq e$. Here, the implicit $O$-constant depends on $K$, and

$$
c_{K}:=\frac{9^{q}}{4 \cdot 6 !}\left(\frac{2^{r}(2 \pi)^{s}}{\sqrt{\left|\Delta_{K}\right|}}\right)^{9}\left(\frac{h_{K} R_{K}}{\omega_{K}}\right)^{7} \prod_{\mathfrak{p}}\left(1-\frac{1}{\mathfrak{N p}}\right)^{7}\left(1+\frac{7}{\mathfrak{N p}}+\frac{1}{\mathfrak{N p}^{2}}\right)
$$

where the product runs over all nonzero prime ideals $\mathfrak{p}$ of $\mathfrak{O}_{K}$.

The leading constant. Let us check the leading constant $c_{K}$ in Theorem 1 against the expected one. According to [Batyrev and Tschinkel 1998b, Section 3.4, Step 4], it should have the form

$$
\frac{\gamma_{K^{-1}}(U) \delta_{\mathscr{K}^{-1}}(U) \tau_{\mathscr{K}^{-1}}(U)}{6 !}
$$

where $\gamma_{\mathscr{K}^{-1}}(U)$ is the volume of a certain polytope depending only on $U, \delta_{\mathscr{K}^{-1}}(U)$ is a cohomological invariant, and $\tau_{\mathscr{K}^{-1}}(U)$ is a generalized version of the Tamagawa number introduced by Peyre [1995] for smooth Fano varieties.

Derenthal and Janda [2013, Section 3] computed these constants for our $U$ over arbitrary number fields $K$, using a minimal desingularization $\tilde{S}$ of $S$ constructed 
by blow-ups of $\mathbb{P}^{2}$ in six rational points: We have $\delta_{\mathscr{K}^{-1}}(U)=1$, and, as already given in [Batyrev and Tschinkel 1998b, Section 5.3], $\gamma_{\varkappa^{-1}}(U)=\frac{1}{36}$. The Tamagawa number $\tau_{\mathscr{K}^{-1}}(U)$ is an adelic invariant given as a product of local densities with certain convergence factors

$$
\tau_{\mathscr{K}^{-1}}(U)=\left(\frac{2^{r}(2 \pi)^{s} h_{K} R_{K}}{\omega_{K} \sqrt{\left|\Delta_{K}\right|}}\right)^{7}\left|\Delta_{K}\right|^{-1} \prod_{\nu \mid \infty} \omega_{\mathscr{K}^{-1}, v}\left(\tilde{S}\left(K_{\nu}\right)\right) \prod_{\nu \nmid \infty} \lambda_{\nu}^{-1} \omega_{\mathscr{K}^{-1}, v}\left(\tilde{S}\left(K_{\nu}\right)\right) .
$$

For the Archimedean densities, we have

$$
\omega_{\mathscr{K}^{-1}, v}\left(\tilde{S}\left(K_{v}\right)\right)= \begin{cases}36 & \text { if } K_{v}=\mathbb{R} \\ 36 \pi^{2} & \text { if } K_{v}=\mathbb{C}\end{cases}
$$

The non-Archimedean density at the place $v$ corresponding to the prime ideal $\mathfrak{p}$ of $\mathrm{O}_{K}$ is given by

$$
\lambda_{v}^{-1} \omega_{\mathscr{K}}{ }^{-1}, v\left(\tilde{S}\left(K_{\nu}\right)\right)=\left(1-\frac{1}{\mathfrak{N p}}\right)^{7}\left(1+\frac{7}{\mathfrak{N p}}+\frac{1}{\mathfrak{N p}^{2}}\right) .
$$

Putting this together, we see that the constant $c_{K}$ in Theorem 1 is as expected.

More notation. The ideal class of a nonzero fractional ideal $\mathfrak{a}$ of $K$ is denoted by [a]. We write $P_{K}$ for the group of nonzero principal fractional ideals of $K$. We denote the real embeddings by $\sigma_{1}, \ldots, \sigma_{r}: K \rightarrow \mathbb{R}$ and the complex embeddings by $\sigma_{r+1}, \bar{\sigma}_{r+1}, \ldots, \sigma_{r+s}, \bar{\sigma}_{r+s}: K \rightarrow \mathbb{C}$. The componentwise continuation of $\sigma_{i}$ to $K^{n}$ is also denoted by $\sigma_{i}$. If $v$ is the place corresponding to $\sigma_{i}$ then we put $d_{i}:=d_{\nu}$. When convenient, we write $\alpha^{(i)}:=\sigma_{i}(\alpha)$ for $\alpha \in K$. If $\mathfrak{a}, \mathfrak{b}$ are fractional ideals of $K$, we put $(\mathfrak{a}, \mathfrak{b}):=\mathfrak{a}+\mathfrak{b}$. For any point $\boldsymbol{x}=\left(x_{0}, \ldots, x_{n}\right) \in K^{n+1}$, let $\mathfrak{J}(\boldsymbol{x}):=\left(x_{0} \mathfrak{O}_{K}, \ldots, x_{n} \mathfrak{O}_{K}\right)$. Then, for $\boldsymbol{x} \in K^{4}$,

$$
H(\boldsymbol{x})=\mathfrak{N} \mathfrak{J}(\boldsymbol{x})^{-1} \prod_{i=1}^{r+s} \max \left\{\left|x_{0}^{(i)}\right|,\left|x_{1}^{(i)}\right|,\left|x_{2}^{(i)}\right|,\left|x_{3}^{(i)}\right|\right\}^{d_{i}} .
$$

We fix, once and for all, a system of fundamental units of $\mathscr{O}_{K}$, and denote by $\mathscr{F}$ the multiplicative subgroup of $K^{\times}$generated by this system. Then $\mathscr{F}$ is a free Abelian group of rank $q$, and the unit group $\mathscr{O}_{K}^{\times}$is the direct product $O_{K}^{\times}=\mu_{K} \mathscr{F}$, where $\mu_{K}$ is the group of roots of unity in $K$.

Moreover, we fix, once and for all, a system $\mathscr{b}$ of integral representatives for the ideal classes of $\mathrm{O}_{K}$, that is, a set of $h_{K}$ nonzero ideals of $O_{K}$, one from every ideal class.

\section{Passing to a universal torsor}

In this section, we find a parametrization of the rational points of bounded height on $U$ by (almost) integral points on an open subset of $\mathbb{A}_{K}^{9}$, subject to some height- and 
coprimality conditions, and up to a certain action of $\left(0_{K}^{\times}\right)^{7}$. This parametrization has the merit that, due to the coprimality conditions, the non-Archimedean parts of the height conditions are trivial.

Over $\mathbb{Q}$ and imaginary quadratic number fields, the action of $\left(\mathcal{O}_{K}^{\times}\right)^{7}$ makes no problems, since then $\mathbb{O}_{K}^{\times}$is finite. In general, that is not the case; this is one of the main difficulties which we have to overcome.

While we will use purely number-theoretic arguments, we mention that the open subset of $A^{9}$ is a universal torsor over $S$, and that our construction is motivated by geometric considerations; see [Derenthal and Janda 2013]. The choice of indices might seem slightly counterintuitive at the beginning. It is, however, closely related to those geometric considerations and will lead to a rather symmetric result.

Parametrization. Let $\Psi_{0}: K^{3} \rightarrow K^{4}$ be given by

$$
\Psi_{0}\left(x_{23}, x_{31}, x_{12}\right)=\left(x_{12} x_{23} x_{31}, x_{12} x_{31}^{2}, x_{23} x_{12}^{2}, x_{31} x_{23}^{2}\right) \text {. }
$$

We will also consider $\Psi_{0}$ as a rational map $\mathbb{P}^{2}-\rightarrow \mathbb{P}^{3}$. Let $W \subseteq \mathbb{P}^{2}$ be the open subset

$$
W=\left\{\left(x_{23}: x_{31}: x_{12}\right) \in \mathbb{P}^{2} \mid x_{12} x_{23} x_{31} \neq 0\right\} \text {. }
$$

Then $\Psi_{0}$ induces a bijection between $W(K) \subseteq \mathbb{P}_{2}(K)$ and $U(K) \subseteq \mathbb{P}_{3}(K)$ with inverse $\left(x_{0}: x_{1}: x_{2}: x_{3}\right) \mapsto\left(x_{0}^{2}: x_{0} x_{1}: x_{1} x_{2}\right)$. Therefore,

$$
N(B)=\left|\left\{\boldsymbol{x} \in W(K) \mid H\left(\Psi_{0}(\boldsymbol{x})\right) \leq B\right\}\right| .
$$

Whenever indices $j, k, l$ appear in an expression, this expression is understood to hold for all $(j, k, l) \in\{(1,2,3),(2,3,1),(3,1,2)\}=: A$.

Lemma 2.1. Let $\mathfrak{b}_{1}, \mathfrak{b}_{2}, \mathfrak{b}_{3}$ be nonzero ideals of $\mathfrak{O}_{K}$, and let $\mathfrak{c}:=\left(\mathfrak{b}_{1}, \mathfrak{b}_{2}, \mathfrak{b}_{3}\right)$. Then there exist unique nonzero ideals $\mathfrak{a}_{1}, \mathfrak{a}_{2}, \mathfrak{a}_{3}, \mathfrak{a}_{12}, \mathfrak{a}_{21}, \mathfrak{a}_{23}, \mathfrak{a}_{32}, \mathfrak{a}_{31}, \mathfrak{a}_{13}$ of $\mathfrak{O}_{K}$ such that

$$
\mathfrak{b}_{j}=\mathfrak{c} \cdot \mathfrak{a}_{j k} \cdot \mathfrak{a}_{k}^{2} \cdot \mathfrak{a}_{l k} \cdot \mathfrak{a}_{j} \cdot \mathfrak{a}_{k j}
$$

and such that the following coprimality conditions hold:

$$
\begin{array}{rllll}
\left(\mathfrak{a}_{k}, \mathfrak{a}_{j}\right)=\mathfrak{O}_{K}, & (2-3) & \left(\mathfrak{a}_{k}, \mathfrak{a}_{l j}\right)=\mathscr{O}_{K}, & (2-6) & \left(\mathfrak{a}_{l k}, \mathfrak{a}_{l j}\right)=\mathfrak{O}_{K}, \\
\left(\mathfrak{a}_{k}, \mathfrak{a}_{k j}\right)=\mathfrak{O}_{K}, & (2-4) & \left(\mathfrak{a}_{k}, \mathfrak{a}_{k l}\right)=\mathfrak{O}_{K}, & (2-7) & \left(\mathfrak{a}_{l k}, \mathfrak{a}_{j l}\right)=\mathfrak{O}_{K}, \\
\left(\mathfrak{a}_{k}, \mathfrak{a}_{j l}\right)=\mathfrak{O}_{K}, & (2-5) & \left(\mathfrak{a}_{l k}, \mathfrak{a}_{j k}\right)=\mathfrak{O}_{K}, & (2-8) & \left(\mathfrak{a}_{j k}, \mathfrak{a}_{k l}\right)=\mathfrak{O}_{K} .
\end{array}
$$

Conversely, given ideals $\mathfrak{a}_{k}, \mathfrak{a}_{j k}, \mathfrak{a}_{l k}$ as in (2-3)-(2-11), the ideals $\mathfrak{b}_{j}$ defined by (2-2) satisfy $\left(\mathfrak{b}_{1}, \mathfrak{b}_{2}, \mathfrak{b}_{3}\right)=\mathfrak{c}$. 
Proof. It is enough to prove the lemma if $\mathfrak{c}=\mathrm{O}_{K}$, since we can always replace $\mathfrak{b}_{j}$ by $\mathfrak{c}^{-1} \mathfrak{b}_{j}$. In this case, we have $\left(\mathfrak{b}_{j}, \mathfrak{b}_{k}^{2}\right)\left(\mathfrak{b}_{l}, \mathfrak{b}_{j}\right) \mid \mathfrak{b}_{j}$. Let

$$
\mathfrak{a}_{j k}:=\frac{\mathfrak{b}_{j}}{\left(\mathfrak{b}_{j}, \mathfrak{b}_{k}^{2}\right)\left(\mathfrak{b}_{l}, \mathfrak{b}_{j}\right)}, \quad \mathfrak{a}_{k}:=\left(\frac{\mathfrak{b}_{j}}{\left(\mathfrak{b}_{j}, \mathfrak{b}_{k}\right)}, \mathfrak{b}_{k}\right), \quad \text { and } \quad \mathfrak{a}_{l k}:=\frac{\left(\mathfrak{b}_{j}, \mathfrak{b}_{k}\right)}{\mathfrak{a}_{k}}
$$

Then the $\mathfrak{a}_{j k}, \mathfrak{a}_{k}, \mathfrak{a}_{l k}$ are nonzero ideals of $\mathfrak{O}_{K}$ and (2-2) holds, since

$$
\left(\mathfrak{b}_{j}, \mathfrak{b}_{k}^{2}\right)=\left(\mathfrak{b}_{j}, \mathfrak{b}_{k}\right) \mathfrak{a}_{k}=\mathfrak{a}_{k}^{2} \mathfrak{a}_{l k} \quad \text { and } \quad\left(\mathfrak{b}_{l}, \mathfrak{b}_{j}\right)=\mathfrak{a}_{j} \mathfrak{a}_{k j} .
$$

One readily verifies that the left-hand sides in conditions (2-3)-(2-6), (2-9), and (2-10) divide $\left(\mathfrak{b}_{1}, \mathfrak{b}_{2}, \mathfrak{b}_{3}\right)=\mathfrak{O}_{K}$. Similarly, the left-hand sides in (2-7), (2-11) divide $\left(\mathfrak{b}_{j} /\left(\mathfrak{b}_{j}, \mathfrak{b}_{k}\right), \mathfrak{b}_{k} /\left(\mathfrak{b}_{j}, \mathfrak{b}_{k}\right)\right)=\mathfrak{O}_{K}$, and the left-hand side in (2-8) divides $\left(\mathfrak{b}_{k} / \mathfrak{a}_{k}, \mathfrak{b}_{j} /\left(\left(\mathfrak{b}_{j}, \mathfrak{b}_{k}\right) \mathfrak{a}_{k}\right)\right)=\mathfrak{O}_{K}$

Now assume that (2-2) holds, with given nonzero ideals $\mathfrak{a}_{k}, \mathfrak{a}_{j k}, \mathfrak{a}_{l k}$ satisfying the coprimality conditions (2-3)-(2-11). These conditions imply that $\left(\mathfrak{b}_{j}, \mathfrak{b}_{k}\right)=\mathfrak{a}_{k} \mathfrak{a}_{l k}$, and furthermore $\left(\mathfrak{b}_{j} /\left(\mathfrak{a}_{k} \mathfrak{a}_{l k}\right), \mathfrak{b}_{k}\right)=\mathfrak{a}_{k}$. Thus, the $\mathfrak{a}_{k}, \mathfrak{a}_{l k}$ are as in (2-12). Clearly, this holds as well for the $\mathfrak{a}_{j k}$, and uniqueness is proved.

The last assertion is again a direct consequence of (2-3)-(2-11).

The coprimality conditions (2-3)-(2-11) can be expressed in a more convenient way: Let $G=(V, E)$ be the graph with vertex set $V:=\{1,2,3,12,21,23,32,31,13\}$ and edge set $E:=\{\{k, j k\},\{k, l k\},\{k l, l k\} \mid(j, k, l) \in A\}$. We can draw it as follows:

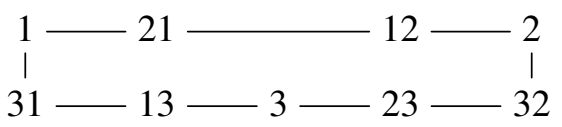

Then (2-3)-(2-11) hold if and only if $\left(\mathfrak{a}_{v}, \mathfrak{a}_{w}\right)=\mathfrak{O}_{K}$ for all pairs $(v, w)$ of nonadjacent vertices of $V$. If we denote the edge set of the complement graph by $E^{\prime}$, this means that

$$
\text { for any }\{v, w\} \in E^{\prime} \text {, we have }\left(\mathfrak{a}_{v}, \mathfrak{a}_{w}\right)=\mathscr{O}_{K} \text {. }
$$

For every point $\left(x_{23}: x_{31}: x_{12}\right) \in W(K)$, the ideal class $\left[\mathfrak{J}\left(x_{23}, x_{31}, x_{12}\right)\right]$ is welldefined, and $\left[\mathfrak{J}\left(x_{23}, x_{31}, x_{12}\right)\right]=[C]$, for some $C \in \mathscr{C}$. By multiplying with a suitable element of $K^{\times}$, we can choose a representative $\boldsymbol{x}=\left(x_{23}, x_{31}, x_{12}\right) \in\left(0_{K} \backslash\{0\}\right)^{3}$ with $\mathfrak{J}(\boldsymbol{x})=C$. This representative is unique up to scalar multiplication by units in $\mathbb{O}_{K}^{\times}$.

We apply Lemma 2.1 to the principal ideals $\mathfrak{b}_{j}:=x_{j k} \widehat{O}_{K}$ and obtain

$$
x_{j k} \widehat{O}_{K}=C \cdot \mathfrak{a}_{j k} \cdot \mathfrak{a}_{k}^{2} \cdot \mathfrak{a}_{l k} \cdot \mathfrak{a}_{j} \cdot \mathfrak{a}_{k j},
$$

with unique ideals $\mathfrak{a}_{v}$ of $\mathfrak{O}_{K}$ satisfying (2-13). For all $v \in V \backslash\{12,23,31\}$, there is a unique $C_{v} \in \mathscr{C}$ with $\left[\mathfrak{a}_{v}\right]=\left[C_{v}^{-1}\right]$. Choose $y_{v} \in K^{\times}$with $y_{v} \mathfrak{O}_{K}=\mathfrak{a}_{v} C_{v}$, and 
define $y_{12}, y_{23}, y_{31} \in K^{\times}$by the equations

$$
x_{j k}=y_{j k} \cdot y_{k}^{2} \cdot y_{l k} \cdot y_{j} \cdot y_{k j}
$$

Then

$$
y_{j k} \widehat{O}_{K}=\mathfrak{a}_{j k} C_{j k} \quad \text { with } C_{j k}:=C C_{k}^{-2} C_{l k}^{-1} C_{j}^{-1} C_{k j}^{-1} .
$$

For $C=\left(C, C_{1}, C_{2}, C_{3}, C_{21}, C_{32}, C_{13}\right) \in \mathscr{C}^{7}$, we define $M_{\boldsymbol{C}}$ as the set of all $\boldsymbol{y}=\left(y_{v}\right)_{v \in V} \in\left(K^{\times}\right)^{9}$ such that

$$
y_{v} \in C_{v} \text { for all } v \in V \text {, and the ideals } \mathfrak{a}_{v}:=y_{v} C_{v}^{-1} \text { satisfy (2-13). }
$$

By what we have shown above, relations (2-14) define a surjective mapping

$$
\phi: \bigcup_{\boldsymbol{C} \in \mathscr{C}^{7}} M_{\boldsymbol{C}} \rightarrow W(K) .
$$

If $\boldsymbol{y} \in M_{\boldsymbol{C}}$ and $\phi(\boldsymbol{y})=\left(x_{23}: x_{31}: x_{12}\right)$ with $x_{j k}$ as in (2-14) then

$$
x_{j k} \widehat{O}_{K}=C \cdot \mathfrak{a}_{j k} \cdot \mathfrak{a}_{k}^{2} \cdot \mathfrak{a}_{l k} \cdot \mathfrak{a}_{j} \cdot \mathfrak{a}_{k j} .
$$

By Lemma 2.1, we have $\mathfrak{J}\left(x_{23}, x_{31}, x_{12}\right)=C$, and the $\mathfrak{a}_{v}$ (and thus as well the $C_{v}$ ) are uniquely determined by the $x_{j k} \mathfrak{O}_{K}$. In particular, the sets $M_{C}, C \in \mathscr{C}^{7}$, are pairwise disjoint. Moreover, $\left(x_{23}, x_{31}, x_{12}\right)$ and the $y_{v}, v \in V$, are determined by $\phi(y)$ up to multiplication by units. Therefore, $\phi(y)=\phi(z)$ if and only if there are units $\zeta, \zeta_{v} \in \mathcal{O}_{K}^{\times}$with

$$
z_{v}=\zeta_{v} y_{v} \text { for all } v \in V \text { and } \zeta_{j k} \zeta_{k}^{2} \zeta_{l k} \zeta_{j} \zeta_{k j}=\zeta \text { for all }(j, k, l) \in A .
$$

By eliminating the $\zeta_{j k}$, we see that $\phi(y)=\phi(z)$ if and only if $\boldsymbol{y}$ and $z$ are in the same orbit of the action $\odot$ of $\left(\mathcal{O}_{K}^{\times}\right)^{7}$ on $\left(K^{\times}\right)^{9}$ given by

$$
\left(\zeta, \zeta_{1}, \zeta_{2}, \zeta_{3}, \zeta_{21}, \zeta_{32}, \zeta_{13}\right) \odot\left(y_{v}\right)_{v}:=\left(z_{v}\right)_{v}
$$

where $z_{v}:=\zeta_{v} y_{v}$ for all $v \in V \backslash\{12,23,31\}$ and $z_{j k}:=\zeta \zeta_{k}^{-2} \zeta_{l k}^{-1} \zeta_{j}^{-1} \zeta_{k j}^{-1} y_{j k}$.

In what follows, it will be more convenient to work with the free Abelian subgroup $\mathscr{F}_{\mathrm{F}}$ of $\mathcal{O}_{K}^{\times}$generated by our fixed system of fundamental units. Clearly, $\left(\mathcal{O}_{K}^{\times}\right)^{7}$ is the direct product $\left(\mathbb{O}_{K}^{\times}\right)^{7}=\mu_{K}^{7} \cdot \mathscr{F}^{7}$. Since the action of $\left(\mathcal{O}_{K}^{\times}\right)^{7}$ on $\left(K^{\times}\right)^{9}$ is free, every orbit of $\left(K^{\times}\right)^{9}$ under the action of $\left(0_{K}^{\times}\right)^{7}$ is the union of $\left|\mu_{K}^{7}\right|=\omega_{K}^{7}$ orbits under the action of $\mathscr{F}^{7}$.

Let $\mathscr{R}$ be a system of representatives for the orbits of $\left(K^{\times}\right)^{9}$ under the action of $\mathscr{F}^{7}$. Then $\phi$ induces an $\omega_{K}^{7}$-to-1 map

$$
\phi: \bigcup_{C \in \mathscr{C}^{7}}\left(M_{\boldsymbol{C}} \cap \mathscr{R}\right) \rightarrow W(K) .
$$

The benefits of our construction become apparent in the height condition. With $\boldsymbol{x}=\left(x_{23}, x_{31}, x_{12}\right)$ as in (2-14), we have $\psi_{0}(\boldsymbol{x})=y_{1}^{2} y_{2}^{2} y_{3}^{2} y_{21} y_{32} y_{13} \cdot \psi(\boldsymbol{y})$, where 


$$
\psi(\boldsymbol{y})=\left(\psi(\boldsymbol{y})_{0}, \psi(\boldsymbol{y})_{1}, \psi(\boldsymbol{y})_{2}, \psi(\boldsymbol{y})_{3}\right),
$$

with

$$
\psi(\boldsymbol{y})_{0}:=\prod_{v \in V} y_{v} \quad \text { and } \quad \psi(\boldsymbol{y})_{j}:=y_{j}^{3} y_{j k} y_{j l} y_{k j}^{2} y_{l j}^{2} \quad \text { for } 1 \leq j \leq 3 .
$$

Therefore,

$$
H\left(\psi_{0}(\boldsymbol{x})\right)=H(\psi(\boldsymbol{y}))=\mathfrak{N} \mathfrak{J}(\psi(\boldsymbol{y}))^{-1} \prod_{i=1}^{r+s} \max _{0 \leq j \leq 3}\left\{\left|\psi(\boldsymbol{y})_{j}^{(i)}\right|\right\}^{d_{i}} .
$$

A straightforward computation using $y_{v}=\mathfrak{a}_{v} C_{v}$ and (2-13) shows that

$$
\mathfrak{J}(\psi(\boldsymbol{y}))=C^{3} C_{1}^{-2} C_{2}^{-2} C_{3}^{-2} C_{21}^{-1} C_{32}^{-1} C_{13}^{-1}
$$

By our construction, $\psi(\boldsymbol{y})$ satisfies the equation $\psi(\boldsymbol{y})_{0}^{3}=\psi(\boldsymbol{y})_{1} \psi(\boldsymbol{y})_{2} \psi(\boldsymbol{y})_{3}$. Since this holds as well for all conjugates, the maximum is always one of $\left|\psi(\boldsymbol{y})_{1}^{(i)}\right|$, $\left|\psi(\boldsymbol{y})_{2}^{(i)}\right|,\left|\psi(\boldsymbol{y})_{3}^{(i)}\right|$. We define

$$
\mathscr{R}(B):=\left\{\boldsymbol{y} \in \mathscr{R} \mid \prod_{i=1}^{r+s} \max _{1 \leq j \leq 3}\left\{\left|\sigma_{i}\left(y_{j}^{3} y_{j k} y_{j l} y_{k j}^{2} y_{l j}^{2}\right)\right|\right\}^{d_{i}} \leq B\right\} .
$$

The results of this section can be summarized as follows.

Proposition 2.2. Let $M_{C}$ be as in (2-15), let $\Re$ be any system of representatives for the orbits of $\left(K^{\times}\right)^{9}$ under the action $\odot$ of $\mathscr{F}^{7}$ given by (2-16), and let $\mathscr{R}(B)$ be as in (2-17). Then $M_{C} \cap \mathscr{R}(B)$ is finite for all $B>0, C \in \mathscr{C}^{7}$, and

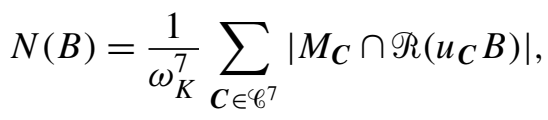

where $u_{C}:=\mathfrak{N}\left(C^{3} C_{1}^{-2} C_{2}^{-2} C_{3}^{-2} C_{21}^{-1} C_{32}^{-1} C_{13}^{-1}\right)$.

A system of representatives for the orbits. We construct a system $\mathscr{R}$ of representatives for the orbits of $\left(K^{\times}\right)^{9}$ under the action $\odot$ of $\mathscr{F}^{7}$ given by (2-16).

Lemma 2.3. Let $\alpha_{1}, \alpha_{2}, \alpha_{3} \in \mathscr{F}$ and consider the system of equations

$$
\zeta \zeta_{k}^{-2} \zeta_{j}^{-1}=\alpha_{j}, \text { for }(j, k) \in\{(1,2),(2,3),(3,1)\},
$$

with variables $\zeta, \zeta_{j} \in \mathscr{F}_{\text {. }}$

(i) If $\alpha_{1} \alpha_{2} \alpha_{3}$ is not a cube in $\mathscr{F}$ then this system has no solutions.

(ii) If $\alpha_{1} \alpha_{2} \alpha_{3}=\xi^{3}$ with $\xi \in \mathscr{F}$ then the solutions are given by

$$
\zeta_{1}=\delta, \quad \zeta_{2}=\delta \xi^{-1} \alpha_{3}, \quad \zeta_{3}=\delta \xi \alpha_{2}^{-1}, \quad \zeta=\delta^{3} \xi \alpha_{2}^{-1} \alpha_{3},
$$

for all $\delta \in \mathscr{F}$. 
Proof. Equations (2-18) imply that

$$
\zeta^{3} \zeta_{j}^{-9}=\alpha_{j} \alpha_{k}^{-2} \alpha_{l}^{4}=\alpha_{1} \alpha_{2} \alpha_{3} \alpha_{k}^{-3} \alpha_{l}^{3},
$$

which proves (i).

Now assume that $\alpha_{1} \alpha_{2} \alpha_{3}=\xi^{3}$ for some $\xi \in \mathscr{F}$. Then $\xi$ is unique since $\mathscr{F}$ is free Abelian. Direct computations verify that the values given in (ii) are solutions.

Given any solution $\left(\zeta, \zeta_{1}, \zeta_{2}, \zeta_{3}\right)$ of (2-18), let $\delta:=\zeta_{1}$. Then (2-19) with $j=1$ shows that $\zeta$ has the desired form. Similar computations using (2-19) with $j=2$ and $j=3$ prove that $\zeta_{2}$ and $\zeta_{3}$ are as desired.

Let $H$ be the subgroup of $\left(K^{\times}\right)^{6}$ of all $\underline{\alpha}=\left(\alpha_{12}, \alpha_{21}, \alpha_{23}, \alpha_{32}, \alpha_{31}, \alpha_{13}\right) \in \mathscr{F}^{6}$ for which $\alpha_{12} \alpha_{21}^{2} \alpha_{23} \alpha_{32}^{2} \alpha_{31} \alpha_{13}^{2}$ is a cube in $\mathscr{F}$.

Lemma 2.4. Let $\mathscr{R}_{1} \subseteq\left(K^{\times}\right)^{3}$ be a system of representatives for the orbits of $\left(K^{\times}\right)^{3}$ under the action of $\mathscr{F}_{F}$ by scalar multiplication, and let $\mathscr{R}_{2} \subseteq\left(K^{\times}\right)^{6}$ be a system of representatives for $\left(K^{\times}\right)^{6} / H$. Then $\mathscr{R}:=\mathscr{R}_{1} \times \mathscr{R}_{2}$ is a system of representatives for the orbits of $\left(K^{\times}\right)^{9}$ under the action $\odot$ of $\mathscr{F}^{7}$.

Proof. Let $\boldsymbol{y}=\left(y_{v}\right)_{v \in V} \in\left(K^{\times}\right)^{9}$. Then there is a unique $\underline{\alpha} \in H$ such that

$$
\left(\alpha_{12} y_{12}, \alpha_{21} y_{21}, \alpha_{23} y_{23}, \alpha_{32} y_{32}, \alpha_{31} y_{31}, \alpha_{13} y_{13}\right) \in \mathscr{R}_{2} \text {. }
$$

The elements $\underline{\zeta}=\left(\zeta, \zeta_{1}, \zeta_{2}, \zeta_{3}, \zeta_{21}, \zeta_{32}, \zeta_{13}\right) \in \mathscr{F}^{7}$ with $\underline{\zeta} \odot \boldsymbol{y} \in\left(K^{\times}\right)^{3} \times \mathscr{R}_{2}$ are those satisfying

$$
\zeta_{k j}=\alpha_{k j} \text { and } \zeta \zeta_{k}^{-2} \zeta_{l k}^{-1} \zeta_{j}^{-1} \zeta_{k j}^{-1}=\alpha_{j k}
$$

With $\alpha_{j}:=\alpha_{j k} \alpha_{k j} \alpha_{l k}$, this simplifies to (2-18). Now

$$
\alpha_{1} \alpha_{2} \alpha_{3}=\alpha_{12} \alpha_{21}^{2} \alpha_{23} \alpha_{32}^{2} \alpha_{31} \alpha_{13}^{2}
$$

is a cube in $\mathscr{F}_{\text {}}$, so $\zeta, \zeta_{1}, \zeta_{2}, \zeta_{3}$ are of the form given in Lemma 2.3(ii), for $\delta \in$ $\mathscr{F}_{F}$. There is exactly one $\delta \in \mathscr{F}$ such that the corresponding $\zeta_{1}, \zeta_{2}, \zeta_{3}$ satisfy $\left(\zeta_{1} y_{1}, \zeta_{2} y_{2}, \zeta_{3} y_{3}\right) \in \mathscr{R}_{1}$. Hence, there is exactly one $\underline{\zeta} \in \mathscr{F}^{7}$ with $\underline{\zeta} \odot \boldsymbol{y} \in \mathscr{R}$.

Lemma 2.5. Let $R \subseteq K^{\times}$be a system of representatives for $K^{\times} / \mathscr{F}$, and let $R_{\mathscr{F}} \subseteq \mathscr{F}_{\mathscr{F}}$ be a system of representatives for $\mathscr{F} /\left\{\xi^{3} \mid \xi \in \mathscr{F}\right\}$. Then

$$
\mathscr{R}_{2}:=\bigcup_{\rho \in R_{\mathscr{F}}}(\rho R \times R \times R \times R \times R \times R)
$$

is a system of representatives for $\left(K^{\times}\right)^{6} / H$.

Proof. Clearly, $\bigcup_{\rho \in \mathscr{R}_{\mathscr{F}}} \rho R$ is a system of representatives for $K^{\times} /\left\{\xi^{3} \mid \xi \in \mathscr{F}^{3}\right\}$. Let $\boldsymbol{y} \in\left(K^{\times}\right)^{6}$. For all $v \in\{21,23,32,31,13\}$, there is exactly one $\alpha_{v} \in \mathscr{F}$ with $\alpha_{v} y_{v} \in R$. Moreover, there is exactly one $\xi \in \mathscr{F}$ such that

$$
y_{12}\left(\alpha_{21}^{2} \alpha_{23} \alpha_{32}^{2} \alpha_{31} \alpha_{13}^{2}\right)^{-1} \xi^{3} \in \bigcup_{\rho \in \mathscr{R}_{\mathscr{F}}} \rho R .
$$


Hence, there is exactly one $\alpha_{12}:=\left(\alpha_{21}^{2} \alpha_{23} \alpha_{32}^{2} \alpha_{31} \alpha_{13}^{2}\right)^{-1} \xi^{3} \in \mathscr{F}_{\mathrm{F}}$ such that

$$
\underline{\alpha}=\left(\alpha_{12}, \alpha_{21}, \alpha_{23}, \alpha_{32}, \alpha_{31}, \alpha_{13}\right) \in H \quad \text { and } \quad \underline{\alpha} \boldsymbol{y} \in \mathscr{R}_{2} .
$$

We choose the system $\mathscr{R}=\mathscr{R}_{1} \times \mathscr{R}_{2}$ as in Lemma 2.4 , where $\mathscr{R}_{1}$ is any system of representatives for the diagonal action of $\mathscr{F}$ on $\left(K^{\times}\right)^{3}$, and $\mathscr{R}_{2}$ is as in Lemma 2.5.

\section{Proof of Theorem 1}

This section is a generalization of [Derenthal and Janda 2013, Section 5]. We reduce Theorem 1 to a central lemma (Lemma 3.1), whose proof will take up the rest of the article. We assume that $K$ is of degree $d \geq 2$. Over $\mathbb{Q}$, one would need to replace Lemma 5.2 by a slightly more intricate argument to make the sum over the error terms converge, for which we refer to [Heath-Brown and Moroz 1999].

Möbius inversions. Let $\boldsymbol{C}=\left(C, C_{1}, C_{2}, C_{3}, C_{21}, C_{32}, C_{13}\right) \in \mathscr{C}^{7}$ be fixed. We investigate the quantity $\left|M_{C} \cap \Re_{(}\left(u_{C} B\right)\right|$ from Proposition 2.2. We can write

$$
\left|M_{C} \cap \Re_{(}\left(u_{C} B\right)\right|=\sum_{\substack{y \in \mathscr{R}_{(}\left(u_{C} B\right) \\(2-15) \text { holds }}} 1 .
$$

Möbius inversion for all the coprimality conditions in (2-13) yields

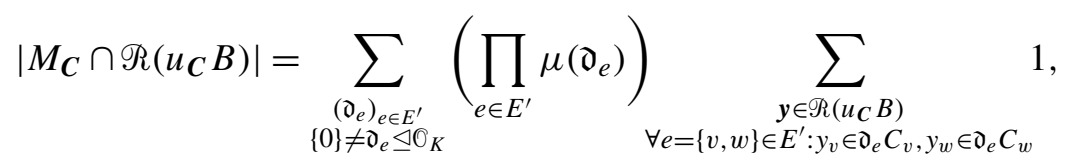

where each $\mathfrak{d}_{e}$ runs over all nonzero ideals of $\mathscr{O}_{K}$ and $\mu$ is the Möbius function for nonzero ideals of $O_{K}$. Lemma 3.1 will imply that the last sum is always finite and nonzero for at most finitely many $\left(\mathfrak{d}_{e}\right)_{e \in E^{\prime}}$. With $\mathfrak{a}_{v}:=\bigcap_{v \in e \in E^{\prime}} \mathfrak{d}_{e} C_{v}$, we obtain

$$
\sum_{\substack{\boldsymbol{y} \in \mathscr{R}\left(u_{C} B\right) \\ \forall e=\{v, w\} \in E^{\prime}: y_{v} \in \mathfrak{d}_{e} C_{v}, y_{w} \in \mathfrak{d}_{e} C_{w}}} 1=\sum_{\substack{\boldsymbol{y} \in \mathscr{R}\left(u_{C} B\right) \\ \forall v: y_{v} \in \mathfrak{a}_{v}}} 1 .
$$

We estimate this sum by the following lemma. Its proof is central to this article and will be given in Section 5 .

Lemma 3.1. For every $v \in V$, let $\mathfrak{a}_{v}$ be a fractional ideal of $K$ with $\mathfrak{N a}_{v} \geq c$, for some constant $c>0$ depending only on $K$. With $\mathscr{R}(B)$ as in (2-17), we have

$$
\begin{aligned}
& \sum_{\substack{y \in \mathscr{R}(B) \\
\forall v: y_{v} \in \mathfrak{a}_{v}}} 1=\frac{9^{q}}{4 \cdot 6 !}\left(\frac{2^{r}(2 \pi)^{s}}{\sqrt{\left|\Delta_{K}\right|}}\right)^{9} \frac{R_{K}^{7}}{\prod_{v \in V} \mathfrak{N a}_{v}} B(\log B)^{6} \\
& \quad+O\left(\frac{\max _{j}\left\{\mathfrak{N a}_{j}\right\}^{1 / d}}{\prod_{j} \mathfrak{N a}_{j} \prod_{i \neq j} \mathfrak{N a}_{i j}^{1-2 /(3 d)}} B(\log B)^{5}\right),
\end{aligned}
$$

for $B \geq e$. The implicit $O$-constant depends on $K$. 
For any $\left(\mathfrak{d}_{e}\right)_{e \in E^{\prime}}$ and $v \in V$, we define $r_{v}:=\mathfrak{N}\left(\cap_{v \in e \in E^{\prime}} \mathfrak{d}_{e}\right)$,

$$
R_{1}:=\prod_{v \in V} r_{v}, \quad \text { and } \quad R_{2}:=\max _{j}\left\{r_{j}\right\}^{-1 / d} \prod_{j} r_{j} \prod_{i \neq j} r_{i j}^{1-2 /(3 d)} .
$$

We notice that $\mathfrak{N a}_{v}=\mathfrak{N}\left(\cap_{v \in e \in E^{\prime}} \mathfrak{d}_{e} C_{v}\right)=\mathfrak{N}\left(C_{v}\right) r_{v}$. Recall that we defined $C_{j k}:=$ $C C_{k}^{-2} C_{l k}^{-1} C_{j}^{-1} C_{k j}^{-1}$ for $j k \in\{12,23,31\}$, so

$$
\prod_{v \in V} \mathfrak{N} C_{v}=\mathfrak{N}\left(C^{3} C_{1}^{-2} C_{2}^{-2} C_{3}^{-2} C_{21}^{-1} C_{32}^{-1} C_{13}^{-1}\right)=u_{C} .
$$

Since the $C, C_{j}, C_{k j}$ are members of the fixed finite set $\mathscr{C}$, their absolute norms are bounded from below and above by positive constants depending only on $K$. With this and Lemma 3.1, we obtain

$$
\sum_{\substack{y \in \mathscr{R}_{(}\left(u_{C} B\right) \\ y_{v} \in \mathfrak{a}_{v}}} 1=\frac{9^{q}}{4 \cdot 6 !}\left(\frac{2^{r}(2 \pi)^{s}}{\sqrt{\left|\Delta_{K}\right|}}\right)^{9} R_{K}^{7} \frac{B}{R_{1}}(\log B)^{6}+O\left(\frac{B}{R_{2}}(\log B)^{5}\right),
$$

whenever $B \geq e / u_{C}$. Otherwise, the error term dominates the main term. Let

$$
\omega:=\sum_{\substack{\left(\mathfrak{d}_{e}\right)_{e \in E^{\prime}} \\\{0\} \neq \mathfrak{d}_{\mathfrak{e}} \unlhd \mathbb{O}_{K}}} \prod_{e \in E^{\prime}} \mu\left(\mathfrak{d}_{e}\right) R_{1}^{-1}, \quad \rho:=\sum_{\substack{\left(\mathfrak{d}_{e} e_{e \in E^{\prime}} \\\{0\} \neq \mathfrak{d}_{\mathfrak{e}} \unlhd \mathbb{O}_{K}\right.}} \prod_{e \in E^{\prime}}\left|\mu\left(\mathfrak{d}_{e}\right)\right| R_{2}^{-1} .
$$

We will see in Lemma 3.2 that these sums converge under our assumption that $d \geq 2$. Since the sum defining $\rho$ converges, (3-1) and (3-2) yield

$$
\left|M_{C} \cap \Re_{R}\left(u_{C} B\right)\right|=\frac{9^{q}}{4 \cdot 6 !}\left(\frac{2^{r}(2 \pi)^{s}}{\sqrt{\left|\Delta_{K}\right|}}\right)^{9} R_{K}^{7} \omega B(\log B)^{6}+O\left(B(\log B)^{5}\right) .
$$

Computation of the constant. We notice that the above expression for

$$
\left|M_{C} \cap \Re_{R}\left(u_{C} B\right)\right|
$$

does not depend on $\boldsymbol{C} \in \mathscr{C}^{7}$. Therefore, Proposition 2.2 implies

$$
N(B)=\frac{9^{q}}{4 \cdot 6 !}\left(\frac{2^{r}(2 \pi)^{s}}{\sqrt{\left|\Delta_{K}\right|}}\right)^{9}\left(\frac{h_{K} R_{K}}{\omega_{K}}\right)^{7} \omega B(\log B)^{6}+O\left(B(\log B)^{5}\right) .
$$

Theorem 1 is an immediate consequence of the following lemma.

Lemma 3.2. Let $\omega, \rho$ be as in (3-4), with $R_{1}, R_{2}$ as in (3-3). If $d \geq 2$ then both sums converge, and

$$
\omega=\prod_{\mathfrak{p}}\left(1-\frac{1}{\mathfrak{N p}}\right)^{7}\left(1+\frac{7}{\mathfrak{N p}}+\frac{1}{\mathfrak{N p}^{2}}\right)
$$

where the product runs over all nonzero prime ideals $\mathfrak{p}$ of $\mathfrak{O}_{K}$. 
Proof. The proof is a straightforward generalization of the one in [Derenthal and Janda 2013, Section 5]. An obvious modification of the argument given there shows that the Euler factor of $\rho$ corresponding to a prime ideal $\mathfrak{p}$ of $\mathrm{O}_{K}$ is $1+O\left(\mathfrak{N p}^{-(6 d-5) /(3 d)}\right)$, so the sum defining $\rho$ is convergent whenever $d \geq 2$. Since $\omega \leq \rho$, the sum defining $\omega$ converges as well.

Let $A(x)$ be the polynomial defined [ibid., Section 5], and $A_{\mathfrak{p}}$ the Euler factor of $\omega$ corresponding to $\mathfrak{p}$. Then we have $A_{\mathfrak{p}}=A\left(\mathfrak{N p}^{-1}\right)$, and (3-5) follows from the investigation of $A(x)$ [ibid., Section 5].

This completes our proof of Theorem 1, up to proving Lemma 3.1.

\section{Auxiliary results}

Let $n, M$ be positive integers and $L>0$. $\operatorname{By} \operatorname{Lip}(n, M, L)$ we denote the set of all subsets $\mathscr{B}$ of $\mathbb{R}^{n}$ for which there exist $M$ maps $\Phi:[0,1]^{n-1} \rightarrow \mathbb{R}^{n}$ satisfying a Lipschitz condition

$$
|\Phi(v)-\Phi(w)| \leq L|v-w|
$$

such that $\mathscr{B}$ is covered by the union of the images of the maps $\Phi$. Here, $|\cdot|$ is the usual Euclidean norm. (The subsets in $\operatorname{Lip}(1, M, L)$ are just those with at most $M$ elements.) We will use the following lemma to bound the error terms when estimating a sum by an integral. Part (i) generalizes an argument used in [Lang 1994, Chapter VI, Theorem 2].

Lemma 4.1. Let $D, \mathscr{B} \subseteq \mathbb{R}^{n}$ be bounded subsets with $\mathscr{B} \in \operatorname{Lip}(n, M, L)$.

(i) Let $\Lambda \subseteq \mathbb{R}^{n}$ be a lattice. Then

$$
|\{\lambda \in \Lambda \mid(\lambda+D) \cap \mathscr{B} \neq \varnothing\}| \ll_{\Lambda, D} M(L+1)^{n-1} .
$$

(ii) If $D, \mathscr{B}$ are compact then $\left\{\boldsymbol{x} \in \mathbb{R}^{n} \mid(x+D) \cap \mathscr{B} \neq \varnothing\right\}$ is measurable and

$$
\operatorname{Vol}\left\{x \in \mathbb{R}^{n} \mid(x+D) \cap \mathscr{B} \neq \varnothing\right\} \ll_{D} M(L+1)^{n-1} .
$$

Proof. For $x \in \mathbb{R}^{n}$, we have $(x+D) \cap \mathscr{B} \neq \varnothing$ if and only if $x \in \mathscr{B}-D$. If $\mathscr{B}$ and $D$ are compact, the set $\mathscr{B}-D$ is compact as well. This proves measurability of the set in (ii).

Let $\Phi:[0,1]^{n-1} \rightarrow \mathbb{R}^{n}$ be one of the $M$ maps with Lipschitz constant $L$ whose images cover $\mathscr{B}$. We split up $[0,1]^{n-1}$ into $L_{1}^{n-1}$ subcubes of side length $1 / L_{1}$, where $L_{1}:=\lfloor L\rfloor+1$. Let $C$ be one of those subcubes. Then $\Phi(C)$ has diameter at most $\sqrt{n-1} L / L_{1} \leq \sqrt{n-1}$, so it is contained in a closed ball $B_{z}(2 \sqrt{n-1})$ of radius $2 \sqrt{n-1}$ centered at some point $z \in \mathbb{R}^{n}$.

Since $D$ is bounded, it is contained in a closed zero-centered ball $B_{0}\left(R_{D}\right)$ of some radius $R_{D}$. Every point $\boldsymbol{x} \in \mathbb{R}^{n}$ with $(\boldsymbol{x}+D) \cap \Phi(C) \neq \varnothing$ satisfies $x \in B_{z}(2 \sqrt{n-1})-B_{0}\left(R_{D}\right)=B_{z}\left(2 \sqrt{n-1}+R_{D}\right)$. 
The number of lattice points in such a ball is finite and can be bounded independently from $z$. Therefore,

$$
|\{\lambda \in \Lambda \mid(\lambda+D) \cap \Phi(C) \neq \varnothing\}| \ll \Lambda, D 1 .
$$

Moreover,

$$
\operatorname{Vol}\left\{x \in \mathbb{R}^{n} \mid(x+D) \cap \Phi(C) \neq \varnothing\right\} \leq \operatorname{Vol} B_{z}\left(2 \sqrt{n-1}+R_{D}\right) \ll_{D} 1
$$

Summing (4-1) and (4-2) over all $C$ and $\Phi$ yields (i) and (ii).

Counting lattice points. We will need to count lattice points in certain bounded subsets of $\mathbb{R}^{n}$ for lattices $\Lambda \subseteq \mathbb{R}^{n}$ of the form $\Lambda=\Lambda_{1} \times \cdots \times \Lambda_{r}$, where each $\Lambda_{i}$ is a lattice in $\mathbb{R}^{n_{i}}$ and $n_{1}+\cdots+n_{r}=n$. Then we have $\operatorname{det}(\Lambda)=\operatorname{det}\left(\Lambda_{1}\right) \cdots \operatorname{det}\left(\Lambda_{r}\right)$, and the successive minima (with respect to the unit ball) of $\Lambda$ are just the successive minima of $\Lambda_{1}, \ldots, \Lambda_{r}$. Several authors (for instance [Christensen and Gubler 2008; Masser and Vaaler 2007]) provide counting results where the first successive minimum is reflected in the error term by making an argument from [Lang 1994, Chapter VI, Theorem 2] explicit. For our application, we need the error term to reflect information about all the lattices $\Lambda_{i}$, which is accomplished with the help of a theorem by Widmer.

Theorem 4.2 [Widmer 2010, Theorem 5.4]. Let $\Lambda$ be a lattice in $\mathbb{R}^{n}$ with successive minima (with respect to the unit ball) $\lambda_{1}, \ldots, \lambda_{n}$. Let $\mathscr{B}$ be a bounded set in $\mathbb{R}^{n}$ with boundary $\partial \mathscr{B} \in \operatorname{Lip}(n, M, L)$. Then $\mathscr{B}$ is measurable, and moreover

$$
|| \Re \cap \Lambda\left|-\frac{\operatorname{Vol} \Re}{\operatorname{det} \Lambda}\right| \leq c_{0}(n) M \max _{0 \leq k<n} \frac{L^{k}}{\lambda_{1} \cdots \lambda_{k}} .
$$

For $k=0$, the expression in the maximum is to be understood as 1 . Furthermore, one can choose $c_{0}(n)=n^{3 n^{2} / 2}$.

Let $\lambda_{i 1} \leq \cdots \leq \lambda_{i n_{i}}$ be the successive minima of $\Lambda_{i}$, and assume that the $\Lambda_{i}$ are ordered in such a way that $\lambda_{11} \leq \lambda_{21} \leq \cdots \leq \lambda_{r 1}$ holds.

Corollary 4.3. Let $\Lambda$ and $\Lambda_{i}$ be as above, and let $\mathscr{B} \subseteq \mathbb{R}^{n}$ be a bounded set with boundary $\partial \mathscr{B} \in \operatorname{Lip}(n, M, L)$. Then $\mathscr{B}$ is measurable and

$$
|| \Re \cap \Lambda\left|-\frac{\operatorname{Vol} \mathscr{B}}{\operatorname{det} \Lambda}\right| \leq c_{0}(n) M \prod_{i=1}^{r-1}\left(\frac{L}{\lambda_{i 1}}+1\right)^{n_{i}}\left(\frac{L}{\lambda_{r 1}}+1\right)^{n_{r}-1} .
$$

Proof. We use Theorem 4.2. Let $\lambda_{1} \leq \cdots \leq \lambda_{n}$ be the successive minima of $\Lambda$, that is, the $\lambda_{i j}$ in correct order. Clearly,

$$
\max _{0 \leq k<n} \frac{L^{k}}{\lambda_{1} \cdots \lambda_{k}} \leq \prod_{j=1}^{n-1}\left(\frac{L}{\lambda_{j}}+1\right) \leq \prod_{i=1}^{r}\left(\frac{L}{\lambda_{i 1}}+1\right)^{n_{i}} /\left(\frac{L}{\lambda_{i_{0}}}+1\right),
$$


where $i_{0}$ is chosen such that $\lambda_{i_{0} n_{i_{0}}}=\lambda_{n}$. The last expression is at most

$$
\prod_{i=1}^{r-1}\left(\frac{L}{\lambda_{i 1}}+1\right)^{n_{i}}\left(\frac{L}{\lambda_{r 1}}+1\right)^{n_{r}-1} .
$$

Lemma 4.4. Let $\Lambda$ and $\Lambda_{i}$ be as above, and let $\mathscr{B} \subseteq \mathbb{R}^{n}$ be contained in a zerocentered ball of radius $R$. Assume, moreover, that $\partial \mathscr{B} \in \operatorname{Lip}(n, M, L)$, and that the following property holds for all $\boldsymbol{x} \in \mathscr{B}$ :

$$
\text { If we write } \boldsymbol{x}=\left(\boldsymbol{x}_{1}, \ldots, \boldsymbol{x}_{r}\right) \text { with } \boldsymbol{x}_{i} \in \mathbb{R}^{n_{i}} \text { then } \boldsymbol{x}_{i} \neq \mathbf{0} \text { for all } i .
$$

Then $\mathscr{P}$ is measurable and, for all $T \geq 0$, we have

$$
|| T \mathscr{\Re} \cap \Lambda\left|-\frac{T^{n} \operatorname{Vol} \mathscr{B}}{\operatorname{det} \Lambda}\right| \ll_{n, M, R, L} \prod_{i=1}^{r-1}\left(\frac{T}{\lambda_{i 1}}\right)^{n_{i}}\left(\frac{T}{\lambda_{r 1}}\right)^{n_{r}-1} .
$$

Proof. By Theorem 4.2, $\mathscr{B}$ is measurable. We start with the case where $T R<\lambda_{r 1}$. Suppose that $\boldsymbol{a}=\left(\boldsymbol{a}_{1}, \ldots, \boldsymbol{a}_{r}\right) \in T \Re \cap \Lambda$. Then $\boldsymbol{a}_{r} \neq \mathbf{0}$ by (4-3). Therefore, $|\boldsymbol{a}| \geq\left|\boldsymbol{a}_{r}\right| \geq \lambda_{r 1}>T R$, so $\boldsymbol{a} \notin T \mathscr{B}$, a contradiction. Hence, $|T \mathscr{B} \cap \Lambda|=0$. Denote by $V_{1}$ the volume of a ball of radius 1 in $\mathbb{R}^{n}$. Then $\operatorname{Vol} \mathscr{B} \leq R^{n} V_{1}$. We denote the successive minima of $\Lambda$ again by $\lambda_{1}, \ldots, \lambda_{n}$. By Minkowski's second theorem we have

$$
\frac{T^{n} \operatorname{Vol} \mathscr{B}}{\operatorname{det} \Lambda} \leq \frac{V_{1} 2^{n}(R T)^{n}}{\lambda_{1} \cdots \lambda_{n} V_{1}} \leq 2^{n} R^{n-1} \prod_{i=1}^{r-1}\left(\frac{T}{\lambda_{i 1}}\right)^{n_{i}}\left(\frac{T}{\lambda_{r 1}}\right)^{n_{r}-1} .
$$

Now assume $T R \geq \lambda_{r 1}$. Clearly, $\operatorname{Vol}(T \mathscr{B})=T^{n} \operatorname{Vol} \mathscr{B}$ and $\partial(T \mathscr{B}) \in \operatorname{Lip}(n, M, T L)$. To finish the proof, we use Corollary 4.3 and observe that

$$
\begin{aligned}
\prod_{i=1}^{r-1}\left(\frac{T L}{\lambda_{i 1}}+1\right)^{n_{i}}\left(\frac{T L}{\lambda_{r 1}}+1\right)^{n_{r}-1} & \leq \prod_{i=1}^{r-1}\left(\frac{T(L+R)}{\lambda_{i 1}}\right)^{n_{i}}\left(\frac{T(L+R)}{\lambda_{r 1}}\right)^{n_{r}-1} \\
& =(L+R)^{n-1} \prod_{i=1}^{r-1}\left(\frac{T}{\lambda_{i 1}}\right)^{n_{i}}\left(\frac{T}{\lambda_{r 1}}\right)^{n_{r}-1} .
\end{aligned}
$$

The basic sets. Here, we describe the sets $\mathscr{B}$ to which Lemma 4.4 will be applied. These sets were introduced in [Schanuel 1979] and, in a more general context, in [Masser and Vaaler 2007]. Our notation is similar to that of the latter. When talking about lattices, volumes, etc., we identify $\mathbb{C}$ with $\mathbb{R}^{2}$.

Let $\Sigma$ be the hyperplane in $\mathbb{R}^{r+s}$ where $x_{1}+\cdots+x_{r+s}=0$. It is well known that the map $l: K^{\times} \rightarrow \mathbb{R}^{r+s}$ defined by $l(\alpha)=\left(d_{1} \log \left|\alpha^{(1)}\right|, \ldots, d_{r+s} \log \left|\alpha^{(r+s)}\right|\right)$ induces a group homomorphism of $\mathcal{O}_{K}^{\times}$onto a lattice in $\Sigma$, with kernel $\mu_{K}$. In particular, $l$ induces a group isomorphism from $\mathscr{F}$ to $l\left(\mathbb{O}_{K}^{\times}\right)$. Let $F$ be a fundamental 
parallelotope for this lattice, and let $\delta:=\left(d_{1}, \ldots, d_{r+s}\right) \in \mathbb{R}^{r+s}$. We define the vector sums

$$
F(\infty):=F+\mathbb{R} \delta \quad \text { and } \quad F(T):=F+(-\infty, \log T] \delta \quad \text { for } T>0 .
$$

Then $F(\infty)$ is a system of representatives for the orbits of the additive action of $l(\mathscr{F})=l\left(\mathcal{O}_{K}^{\times}\right)$on $\mathbb{R}^{r+s}$. Let $S_{F}^{n}(T)$ be the set of all

$$
\left(z_{1,1}, \ldots, z_{1, n}, \ldots, z_{r+s, 1}, \ldots, z_{r+s, n}\right) \in\left(\mathbb{R}^{n} \backslash\{\mathbf{0}\}\right)^{r} \times\left(\mathbb{C}^{n} \backslash\{\mathbf{0}\}\right)^{s}
$$

such that

$$
\left(d_{i} \log \max _{1 \leq j \leq n}\left\{\left|z_{i, j}\right|\right\}\right)_{i=1}^{r+s} \in F(T) .
$$

Since $F \subseteq \Sigma$ and $d_{1}+\cdots+d_{r+s}=d$, this is equivalent to

$$
\left(d_{i} \log \max _{1 \leq j \leq n}\left\{\left|z_{i, j}\right|\right\}\right)_{i=1}^{r+s} \in F(\infty) \quad \text { and } \quad \prod_{i=1}^{r+s} \max _{1 \leq j \leq n}\left\{\left|z_{i, j}\right|\right\}^{d_{i}} \leq T^{d} .
$$

The set $S_{F}^{n}(\infty)$ is defined similarly. Here are some basic properties of $S_{F}^{n}(T)$ :

(i) $S_{F}^{n}(T)=T S_{F}^{n}(1)$ is homogeneously expanding.

(ii) $S_{F}^{n}(1)$ is bounded.

(iii) $\partial S_{F}^{n}(1) \in \operatorname{Lip}\left(n d, M_{n}, L_{n}\right)$ for some $M_{n}, L_{n}$.

(iv) $S_{F}^{n}(1)$ is measurable and $\operatorname{Vol} S_{F}^{n}(1)=n^{q} 2^{n r} \pi^{n s} R_{K}$.

Properties (i), (ii) follow directly from the definition, and (iii), (iv) are immediate consequences of Lemmas 3 and 4 of [Masser and Vaaler 2007]. Strictly speaking, the case $n=1$ is not covered in that paper, but the proofs remain correct without change. We need a slightly modified version: Define

$$
S_{F}^{n *}(T):=S_{F}^{n}(T) \cap\left(\left(\mathbb{R}^{\times}\right)^{n r} \times\left(\mathbb{C}^{\times}\right)^{n s}\right) .
$$

Then (i)-(iv) hold as well for $S_{F}^{n *}(T)$. This is clear for (i), (ii), (iv). For (iii), let $X:=\left(\mathbb{R}^{n r} \times \mathbb{C}^{n s}\right) \backslash\left(\left(\mathbb{R}^{\times}\right)^{n r} \times\left(\mathbb{C}^{\times}\right)^{n s}\right)$. Then $\partial S_{F}^{n *}(1) \subseteq \partial S_{F}^{n}(1) \cup\left(\overline{S_{F}^{n}(1)} \cap X\right)$. Since $\overline{S_{F}^{n}(1)}$ is bounded and $X$ is a union of finitely many proper subspaces, we have $\left(\overline{S_{F}^{n}(1)} \cap X\right) \in \operatorname{Lip}\left(n d, M_{n}^{\prime}, L_{n}^{\prime}\right)$, for suitably chosen $M_{n}^{\prime}, L_{n}^{\prime}$, so

$$
\partial S_{F}^{n *}(1) \in \operatorname{Lip}\left(n d, M_{n}+M_{n}^{\prime}, \max \left\{L_{n}, L_{n}^{\prime}\right\}\right) .
$$

\section{Proof of Lemma 3.1}

Whenever we use Vinogradov's $\ll$ notation, the implicit constant may depend on $K$. Let us start by summing over $y_{1}, y_{2}, y_{3}$, for fixed $y_{j k}, y_{k j}$. Write

$$
V^{\prime}:=V \backslash\{1,2,3\}=\{12,21,23,32,31,13\} .
$$


For any choice of $y_{v}, v \in V^{\prime}$, we define $\xi_{j}:=y_{j k} y_{j l} y_{k j}^{2} y_{l j}^{2}$. The height condition in (2-17) implies that

$$
\left|N\left(y_{j}\right)^{3} N\left(\xi_{j}\right)\right|=\prod_{i=1}^{r+s}\left|\sigma_{i}\left(y_{j}^{3} \xi_{j}\right)\right|^{d_{i}} \leq B
$$

For $y_{j} \in \mathfrak{a}_{j}$, we obtain $\left|N\left(\xi_{j}\right)\right| \leq B\left|N\left(y_{j}\right)\right|^{-3} \leq B \mathfrak{N a} \mathfrak{a}_{j}^{-3}$. By our choice of $\mathscr{R}$ in Lemma 2.4, we can write the sum in Lemma 3.1 as

$$
\sum_{\substack{y \in \mathscr{R}(B) \\ y_{v} \in \mathfrak{a}_{v}}} 1=\sum_{\substack{\left(y_{v}\right)_{v \in V^{\prime} \in \mathfrak{R}_{2}} \\ y_{v} \in \mathfrak{a}_{v} \\ \forall j:\left|N\left(\xi_{j}\right)\right| \leq B \mathfrak{N} \mathfrak{a}_{j}^{-3}}} \sum_{\substack{\left(y_{1}, y_{2}, y_{3}\right) \in \mathscr{R}_{1} \\ y_{j} \in \mathfrak{a}_{j} \\ \prod_{i=1}^{r+s} \max _{j}\left\{\left|\sigma_{i}\left(y_{j}^{3} \xi_{j}\right)\right|\right\}^{d_{i}} \leq B}} 1 .
$$

The first summation. Here, we handle the inner sum in (5-1). The necessary tool is provided in Lemma 5.2.

Lemma 5.1. Let $\mathfrak{a}$ be a fractional ideal of $K$, and let $\tau$ be the linear automorphism of $\mathbb{R}^{r} \times \mathbb{C}^{s}$ (regarded as $\mathbb{R}^{d}$ ) given by $\tau\left(z_{1}, \ldots, z_{r+s}\right)=\left(t_{1} z_{1}, \ldots, t_{r+s} z_{r+s}\right)$, with $t_{1}, \ldots, t_{r+s}>0$. Let $\sigma: K \rightarrow \mathbb{R}^{r} \times \mathbb{C}^{s}$ be the standard embedding. Then $\tau \circ \sigma(\mathfrak{a})$ is a lattice in $\mathbb{R}^{r} \times \mathbb{C}^{s}$ of determinant

$$
\operatorname{det}(\tau \circ \sigma(\mathfrak{a}))=t_{1}^{d_{1}} \cdots t_{r+s}^{d_{r+s}} \cdot 2^{-s} \cdot \mathfrak{N}\left(\mathfrak{a}_{j}\right) \cdot \sqrt{\left|\Delta_{K}\right|}
$$

and first successive minimum $\lambda \geq\left(t_{1}^{d_{1}} \cdots t_{r+s}^{d_{r+s}} \cdot \mathfrak{N a}\right)^{1 / d}$.

Proof. For $d=1$, the lemma is trivial, so we assume $d \geq 2$. Classically, $\sigma(\mathfrak{a})$ is a lattice in $\mathbb{R}^{r} \times \mathbb{C}^{s}$ of determinant $2^{-s} \mathfrak{N}\left(\mathfrak{a}_{j}\right) \sqrt{\left|\Delta_{K}\right|}$. Since $\tau$ is a linear automorphism of determinant $t_{1}^{d_{1}} \cdots t_{r+s}^{d_{r+s}}$, it follows immediately that $\tau \circ \sigma(\mathfrak{a})$ is a lattice with the correct determinant.

For $\lambda$, we slightly generalize the argument in [Masser and Vaaler 2007, Lemma 5] (see also [Widmer 2010, Lemma 9.7]). There is an $\alpha \in \mathfrak{a}$ with $\lambda=|\tau \circ \sigma(\alpha)|$. By the inequality of weighted arithmetic and geometric means, we have

$$
\lambda^{2}=\sum_{i=1}^{r+s}\left|t_{i} \alpha^{(i)}\right|^{2} \geq \frac{1}{2} \sum_{i=1}^{r+s} d_{i}\left|t_{i} \alpha^{(i)}\right|^{2} \geq \frac{d}{2}\left(\prod_{i=1}^{r+s}\left|t_{i} \alpha^{(i)}\right|^{d_{i}}\right)^{\frac{2}{d}} \geq\left(t_{1}^{d_{1}} \cdots t_{r+s}^{d_{r+s}}|N(\alpha)|\right)^{\frac{2}{d}} .
$$

The lemma follows upon noticing that $|N(\alpha)| \geq \mathfrak{N a}$.

Lemma 5.2. Given constants $C_{i j}>0$, for $i \in\{1, \ldots, r+s\}$ and $j \in\{1,2,3\}$, let

$$
C_{j}:=C_{1 j}^{d_{1}} \cdots C_{r+s, j}^{d_{r+s}} .
$$

Let $\mathfrak{a}_{1}, \mathfrak{a}_{2}, \mathfrak{a}_{3} \neq\{0\}$ be fractional ideals of $K$, and $\mathscr{R}_{1}$ a system of representatives 
for the orbits of $\left(K^{\times}\right)^{3}$ under the action of $\mathscr{F}$ by scalar multiplication. Define

$$
M_{1}(T):=\left(\mathfrak{a}_{1} \times \mathfrak{a}_{2} \times \mathfrak{a}_{3}\right) \cap\left\{\left(y_{1}, y_{2}, y_{3}\right) \in \mathscr{R}_{1} \mid \prod_{i=1}^{r+s} \max _{1 \leq j \leq 3}\left\{C_{i j}\left|y_{j}^{(i)}\right|\right\}^{d_{i}} \leq T^{d}\right\} .
$$

Then $M_{1}(T)$ is finite and

$$
\left|M_{1}(T)\right|=\frac{3^{q} 2^{3 r}(2 \pi)^{3 s} R_{K}}{\left(\sqrt{\left|\Delta_{k}\right|}\right)^{3} C_{1} C_{2} C_{3} \mathfrak{N a}_{1} \mathfrak{N a}_{2} \mathfrak{N a}_{3}} T^{3 d}+O\left(\frac{T^{3 d-1} \max _{j}\left\{C_{j} \mathfrak{N a}_{j}\right\}^{1 / d}}{C_{1} C_{2} C_{3} \mathfrak{N a}_{1} \mathfrak{N a}_{2} \mathfrak{N a}_{3}}\right)
$$

for all $T>0$. The implicit $O$-constant depends only on $K$.

Proof. We notice that $\left|M_{1}(T)\right|$ does not depend on the choice of $\mathscr{R}_{1}$, since both $\mathfrak{a}_{1} \times \mathfrak{a}_{2} \times \mathfrak{a}_{3}$ and the height condition are invariant under scalar multiplication of $\left(y_{1}, y_{2}, y_{3}\right)$ by units. Hence, it is enough to prove the lemma with a specific choice of $\mathscr{R}_{1}$, which we construct below.

Let $\sigma: K^{3} \rightarrow \mathbb{R}^{3 r} \times \mathbb{C}^{3 s}$ be the embedding given by $\sigma(\boldsymbol{y})=\left(\sigma_{i}(\boldsymbol{y})\right)_{i=1}^{r+s}$. For $i \in\{1, \ldots, r+s\}$, let $\phi_{i}$ be the linear automorphism of $\mathbb{R}^{3}$ (if $i \leq r$ ) or $\mathbb{C}^{3}$ (if $i>r$ ) given by $\phi_{i}\left(z_{1}, z_{2}, z_{3}\right)=\left(C_{i 1} z_{1}, C_{i 2} z_{2}, C_{i 3} z_{3}\right)$, and let $\phi: \mathbb{R}^{3 r} \times \mathbb{C}^{3 s} \rightarrow \mathbb{R}^{3 r} \times \mathbb{C}^{3 s}$ be the automorphism obtained by applying the $\phi_{i}$ componentwise.

With $S_{F}^{3 *}(T)$ as in (4-4), we define $\mathscr{R}_{1}$ as the set of all $\boldsymbol{y} \in\left(K^{\times}\right)^{3}$ such that $\phi \circ \sigma(\boldsymbol{y}) \in S_{F}^{3 *}(\infty)$. Then $\mathscr{R}_{1}$ is a system of representatives for the orbits of $\left(K^{\times}\right)^{3}$ under the action of $\mathscr{F}$ by scalar multiplication. Indeed, for any $\boldsymbol{y} \in\left(K^{\times}\right)^{3}$ and $\zeta \in \mathscr{F}$, we have

$$
\left(d_{i} \log \max _{1 \leq j \leq 3}\left\{\left|C_{i j} \sigma_{i}\left(\zeta y_{j}\right)\right|\right\}\right)_{i=1}^{r+s}=\left(d_{i} \log \max _{1 \leq j \leq 3}\left\{\left|C_{i j} \sigma_{i}\left(y_{j}\right)\right|\right\}\right)_{i=1}^{r+s}+l(\zeta),
$$

and $F(\infty)$ is a system of representatives for the orbits of the additive action of $l(\mathscr{F})$ on $\mathbb{R}^{r+s}$.

Let $\Lambda:=\phi \circ \sigma\left(\mathfrak{a}_{1} \times \mathfrak{a}_{2} \times \mathfrak{a}_{3}\right)$. Then $\Lambda$ is a lattice in $\mathbb{R}^{3 r} \times \mathbb{C}^{3 s}$, and $\phi \circ \sigma$ induces a one-to-one correspondence between $M_{1}(T)$ and $\Lambda \cap S_{F}^{3 *}(T)$. Therefore,

$$
\left|M_{1}(T)\right|=\left|\Lambda \cap S_{F}^{3 *}(T)\right| .
$$

Since $S_{F}^{3 *}(T)$ is bounded, $M_{1}(T)$ is finite. To simplify the notation, we change the order of coordinates by

$$
\left(z_{11}, z_{12}, z_{13}, \ldots, z_{r+s, 1}, z_{r+s, 2}, z_{r+s, 3}\right) \mapsto\left(z_{11}, \ldots, z_{r+s, 1}, \ldots, z_{13}, \ldots, z_{r+s, 3}\right) .
$$

This way, $\mathbb{R}^{3 r} \times \mathbb{C}^{3 s}$ becomes $\left(\mathbb{R}^{r} \times \mathbb{C}^{s}\right)^{3}$, and $\Lambda$ becomes

$$
\Lambda=\tau_{1} \circ \sigma\left(\mathfrak{a}_{1}\right) \times \tau_{2} \circ \sigma\left(\mathfrak{a}_{2}\right) \times \tau_{3} \circ \sigma\left(\mathfrak{a}_{3}\right),
$$

where $\sigma: K \rightarrow \mathbb{R}^{r} \times \mathbb{C}^{s}$ is the standard embedding given by $\sigma(y)=\left(\sigma_{i}(y)\right)_{i=1}^{r}$ and

$$
\tau_{j}\left(z_{1}, \ldots, z_{r+s}\right):=\left(C_{1 j} z_{1}, \ldots, C_{r+s, j} z_{r+s}\right) .
$$


Each $\Lambda_{j}:=\tau_{j} \circ \sigma\left(\mathfrak{a}_{j}\right)$ is a lattice in $\mathbb{R}^{r} \times \mathbb{C}^{s}=\mathbb{R}^{d}$. Let $\lambda_{j}$ be the first successive minimum of $\Lambda_{j}$. By Lemma 5.1, we have

$$
\operatorname{det} \Lambda=\operatorname{det} \Lambda_{1} \cdot \operatorname{det} \Lambda_{2} \cdot \operatorname{det} \Lambda_{3}=2^{-3 s}\left(\sqrt{\left|\Delta_{K}\right|}\right)^{3} C_{1} C_{2} C_{3} \mathfrak{N a}_{1} \mathfrak{N a}_{2} \mathfrak{N a}_{3}
$$

and $\lambda_{j} \geq\left(C_{j} \mathfrak{N a}_{j}\right)^{1 / d}$. The lemma now follows from (5-2), Lemma 4.4 and the properties of the basic sets discussed on pages 1464-1465.

The inner sum in (5-1) is exactly $\left|M_{1}(T)\right|$ in Lemma 5.2, with

$$
C_{i j}:=\left|\sigma_{i}\left(\xi_{j}\right)\right|^{1 / 3}, \quad C_{j}:=\left|N\left(\xi_{j}\right)\right|^{1 / 3} \quad \text { and } \quad T:=B^{1 /(3 d)} .
$$

Observe that $C_{1} C_{2} C_{3}=\left|N\left(\xi_{1} \xi_{2} \xi_{3}\right)\right|^{1 / 3}=\prod_{v \in V^{\prime}}\left|N\left(y_{v}\right)\right|$. We define

$$
\begin{aligned}
& \mathcal{M}\left(B,\left(\mathfrak{a}_{v}\right)_{v}\right):=\sum_{\substack{\left(y_{v}\right)_{v \in V^{\prime} \in \mathscr{R}_{2}} \\
y_{v} \in \mathfrak{a}_{v} \\
\forall j:\left|N\left(\xi_{j}\right)\right| \leq B \mathfrak{T a} \mathfrak{a}_{j}^{-3}}} \frac{1}{\prod_{v \in V^{\prime}}\left|N\left(y_{v}\right)\right|}, \\
& \mathscr{R}\left(B,\left(\mathfrak{a}_{v}\right)_{v}\right):=\sum_{\substack{\left(y_{v}\right) \\
y_{v} \in \mathfrak{v}^{\prime} \in \mathfrak{R}_{2} \\
y_{v} \in \mathfrak{a}_{v} \\
\forall j:\left|N\left(\xi_{j}\right)\right| \leq B \mathfrak{N} \mathfrak{a}_{j}^{-3}}} \frac{\max _{j}\left\{\left|N\left(\xi_{j}\right)\right|\right\}^{1 /(3 d)}}{\prod_{v \in V^{\prime}}\left|N\left(y_{v}\right)\right|} .
\end{aligned}
$$

Then (5-1) and Lemma 5.2 imply

$$
\begin{aligned}
& \sum_{\substack{y \in \mathscr{R}(B) \\
y_{v} \in \mathfrak{a}_{v}}} 1=\frac{3^{q} 2^{3 r}(2 \pi)^{3 s} R_{K} B}{\left(\sqrt{\left|\Delta_{K}\right|}\right)^{3} \mathfrak{N a}_{1} \mathfrak{N a}_{2} \mathfrak{N a}_{3}} M\left(B,\left(\mathfrak{a}_{v}\right)_{v}\right) \\
& +O\left(\frac{\max _{j}\left\{\mathfrak{N a}_{j}\right\}^{1 / d}}{\mathfrak{N a}_{1} \mathfrak{N a}_{2} \mathfrak{N a}_{3}} B^{1-1 /(3 d)} \mathscr{R}\left(B,\left(\mathfrak{a}_{v}\right)_{v}\right)\right) .
\end{aligned}
$$

Recall that the $\mathfrak{N a}_{v}$ are bounded from below by a positive constant $c$ depending only on $K$. This implies, for example,

$$
\begin{aligned}
& \mathfrak{N}\left(\mathfrak{a}_{j k} \mathfrak{a}_{j l} \mathfrak{a}_{k j}^{2} \mathfrak{a}_{l j}^{2}\right)^{1 /(3 d)} \ll \prod_{v \in V^{\prime}} \mathfrak{N a a _ { v } ^ { 2 / ( 3 d ) } ,} \\
& \mathfrak{N}\left(\mathfrak{a}_{j}^{3} \mathfrak{a}_{j k} \mathfrak{a}_{j l} \mathfrak{a}_{k j}^{2} \mathfrak{a}_{l j}^{2}\right)^{-1} \leq c_{2},
\end{aligned}
$$

for some constant $c_{2} \geq 1$ depending only on $K$.

The error term. With $\mathscr{R}_{2}$ as in Lemma 2.5 , the term $\mathscr{R}\left(B,\left(\mathfrak{a}_{v}\right)_{v}\right)$ has the form

$$
\mathscr{R}\left(B,\left(\mathfrak{a}_{v}\right)_{v}\right)=\sum_{\rho \in R \mathscr{F}} \sum_{\substack{\forall v \neq 12: y_{v} \in R \cap \mathfrak{a}_{v} \\ y_{12} \in \rho R \cap \mathfrak{a}_{12} \\ \forall j:\left|N\left(\xi_{j}\right)\right| \leq B \mathfrak{N a}_{j}^{-3}}} \frac{\max _{j}\left\{\left|N\left(\xi_{j}\right)\right|\right\}^{1 /(3 d)}}{\prod_{v \in V^{\prime}}\left|N\left(y_{v}\right)\right|} .
$$


Both $R$ and $\rho R$ are systems of representatives for $K^{\times} / \mathscr{F}$, so they contain exactly $\omega_{K}$ generators for every nonzero principal fractional ideal of $K$. Let $H_{v}$ be the principal fractional ideal $H_{v}=y_{v} \mathrm{O}_{K}$. The norm condition and the summand in the inner sum depend only on $\left(H_{v}\right)_{v \in V^{\prime}}$. Therefore, the sum does not depend on $\rho$. Since $\left|\mathscr{R}_{\mathscr{F}}\right|=3^{q} \ll 1$, we obtain

$$
\mathscr{R}\left(B,\left(\mathfrak{a}_{v}\right)_{v}\right) \ll \sum_{\substack{\{0\} \neq H_{v} \in P_{K}, v \in V^{\prime} \\ H_{v} \subseteq \mathfrak{a}_{v} \\ \forall j: \mathfrak{N}\left(H_{j k} H_{j l} H_{k j}^{2} H_{l j}^{2}\right) \leq B \mathfrak{N a} \mathfrak{a}_{j}^{-3}}} \frac{\max _{j}\left\{\mathfrak{N}\left(H_{j k} H_{j l} H_{k j}^{2} H_{l j}^{2}\right)\right\}^{1 /(3 d)}}{\prod_{v \in V^{\prime}} \mathfrak{N}\left(H_{v}\right)} .
$$

We replace $H_{v}$ by $H_{v} \mathfrak{a}_{v}^{-1} \unlhd \mathfrak{O}_{K}$ and use (5-6), (5-7) to bound this sum by

$$
\ll \frac{1}{\prod_{v \in V^{\prime}} \mathfrak{N}\left(\mathfrak{a}_{v}\right)^{1-2 /(3 d)}} \sum_{\substack{\{0\} \neq H_{v} \unlhd \cup_{K}, v \in V^{\prime} \\ H_{v} \in\left[\mathfrak{a}_{v}\right]^{-1} \\ \forall j: \mathfrak{N}\left(H_{j k} H_{j l} H_{k j}^{2} H_{l j}^{2}\right) \leq c_{2} B}} \frac{\max _{j}\left\{\mathfrak{N}\left(H_{j k} H_{j l} H_{k j}^{2} H_{l j}^{2}\right)\right\}^{1 /(3 d)}}{\prod_{v \in V^{\prime}} \mathfrak{N}\left(H_{v}\right)} .
$$

Let us denote the above sum by $\mathscr{R}_{1}\left(B,\left(\mathfrak{a}_{v}\right)_{v}\right)$. What follows is a rather straightforward generalization of arguments used by Heath-Brown and Moroz [1999] and Derenthal and Janda [2013]. By symmetry, we may assume that the maximum in the summand is taken for $j=1$. This allows us to bound $\mathscr{R}_{1}\left(B,\left(\mathfrak{a}_{v}\right)_{v}\right)$ by

$$
\begin{aligned}
& \ll \sum_{\substack{\{0\} \neq H_{v} \unlhd \mathbb{O}_{K}, v \in V^{\prime} \\
\forall j: \mathfrak{N}\left(H_{j k} H_{j l} H_{k j}^{2} H_{l j}^{2}\right) \leq c_{2} B}} \frac{1}{\mathfrak{N}\left(H_{12} H_{13}\right)^{1-1 /(3 d)} \mathfrak{N}\left(H_{21} H_{31}\right)^{1-2 /(3 d)} \mathfrak{N}\left(H_{23} H_{32}\right)} \\
& \ll \sum_{\substack{\{0\} \neq H_{i j} \leq \mathbb{O}_{K}, i \neq 1 \\
\mathfrak{N} H_{i j} \leq c_{2} B}} \frac{1}{\mathfrak{N}\left(H_{21} H_{31}\right)^{1-2 /(3 d)} \mathfrak{N}\left(H_{23} H_{32}\right)} \sum_{\substack{\{0\} \neq U \unlhd \mathbb{O}_{K} \\
\mathfrak{N} U \leq u}} \frac{d(U)}{\mathfrak{N} U^{1-1 /(3 d)}},
\end{aligned}
$$

where $u:=c_{2} B \mathfrak{N}\left(H_{21} H_{31}\right)^{-2}$ and $d$ is the divisor function for nonzero ideals.

Lemma 5.3. For $T \geq 1$, we have

$$
\sum_{\substack{\{0\} \neq \mathfrak{a} \leq \mathbb{O}_{K} \\ \mathfrak{N a} \leq T}} \mathfrak{N a}^{\alpha} \ll \begin{cases}T^{\alpha+1} & \text { if }-1<\alpha \leq 0, \\ \max \{1, \log T\} & \text { if } \alpha=-1 .\end{cases}
$$

Proof. This is a straightforward generalization of [Derenthal and Janda 2013, Lemma 4]. The proof uses Abel's summation formula and the well known fact that

$$
\left|\left\{\{0\} \neq \mathfrak{a} \unlhd \mathfrak{O}_{K} \mid \mathfrak{N a} \leq T\right\}\right| \ll T .
$$


In the following computation, the sums run over nonzero ideals of $\mathbb{O}_{K}$. Using Lemma 5.3, we obtain

$$
\begin{aligned}
\sum_{\mathfrak{N} U \leq u} \frac{d(U)}{\mathfrak{N} U^{1-1 /(3 d)}} & =\sum_{\mathfrak{N} U \leq u} \sum_{V \mid U} \mathfrak{N} U^{-1+1 /(3 d)} \\
& =\sum_{\mathfrak{N} V \leq u} \mathfrak{N} V^{-1+1 /(3 d)} \sum_{\mathfrak{N} U \leq u / \mathfrak{N} V} \mathfrak{N} U^{-1+1 /(3 d)} \\
& \ll \sum_{\mathfrak{N} V \leq c_{2} B} \mathfrak{N} V^{-1+1 /(3 d)}(u / \mathfrak{N} V)^{1 /(3 d)} \ll u^{1 /(3 d)} \log B .
\end{aligned}
$$

Therefore,

$$
\begin{aligned}
\mathscr{R}_{1}\left(B,\left(\mathfrak{a}_{v}\right)_{v}\right) & \ll B^{1 /(3 d)} \log B \sum_{\substack{\{0\} \neq H_{i j} \leq \mathbb{O}_{K}, i \neq 1 \\
\mathfrak{N} H_{i j} \leq c_{2} B}} \frac{1}{\mathfrak{N}\left(H_{21} H_{31} H_{23} H_{32}\right)} \\
& \ll B^{1 /(3 d)}(\log B)^{5} .
\end{aligned}
$$

Having estimated $\mathscr{R}_{1}\left(B,\left(\mathfrak{a}_{v}\right)_{v}\right)$ and thus $\mathscr{R}\left(B,\left(\mathfrak{a}_{v}\right)_{v}\right)$, we obtain from (5-5):

$$
\begin{aligned}
& \sum_{\substack{y \in \mathscr{R}(B) \\
y_{v} \in \mathfrak{a}_{v}}} 1=\frac{3^{q} 2^{3 r}(2 \pi)^{3 s} R_{K} B}{\left(\sqrt{\left|\Delta_{K}\right|}\right)^{3} \mathfrak{N a}_{1} \mathfrak{N a}_{2} \mathfrak{N a}_{3}} M\left(B,\left(\mathfrak{a}_{v}\right)_{v}\right) \\
&+O\left(\frac{\max _{j}\left\{\mathfrak{N a}_{j}\right\}^{1 / d}}{\prod_{j} \mathfrak{N a}_{j} \prod_{i \neq j} \mathfrak{N a}_{i j}^{1-2 /(3 d)}} B(\log B)^{5}\right) .
\end{aligned}
$$

The main term. Just as before, we have

$$
\mathcal{M}\left(B,\left(\mathfrak{a}_{v}\right)_{v}\right)=\sum_{\rho \in R \mathscr{F}} \sum_{\substack{\forall v \neq 12: y_{v} \in R \cap \mathfrak{a}_{v} \\ y 12 \in \rho R \cap \mathfrak{a}_{12} \\ \forall j:\left|N\left(\xi_{j}\right)\right| \leq B \mathfrak{N a} \mathfrak{a}_{j}^{-3}}} \frac{1}{\prod_{v \in V^{\prime}}\left|N\left(y_{v}\right)\right|} .
$$

For all $v \in V^{\prime}$, let $\mathfrak{b}_{v} \in \mathscr{C}$ with $\left[\mathfrak{b}_{v}\right]=\left[\mathfrak{a}_{v}\right]$, and $t_{v} \in K^{\times}$with $t_{v} \mathfrak{a}_{v}=\mathfrak{b}_{v}$. Moreover, we define $b_{j}:=\mathfrak{N}\left(\mathfrak{a}_{j}^{3} \mathfrak{a}_{j k} \mathfrak{a}_{j l} \mathfrak{a}_{k j}^{2} \mathfrak{a}_{l j}^{2}\right)^{-1} \mathfrak{N}\left(\mathfrak{b}_{j k} \mathfrak{b}_{j l} \mathfrak{b}_{k j}^{2} \mathfrak{b}_{l j}^{2}\right)$. Then (5-7) implies that

$$
b_{j} \leq c_{3} \text { for all } j \in\{1,2,3\},
$$

with a constant $c_{3} \geq 1$ depending only on $K$. We replace $y_{v}$ by $t_{v} y_{v}$ and obtain

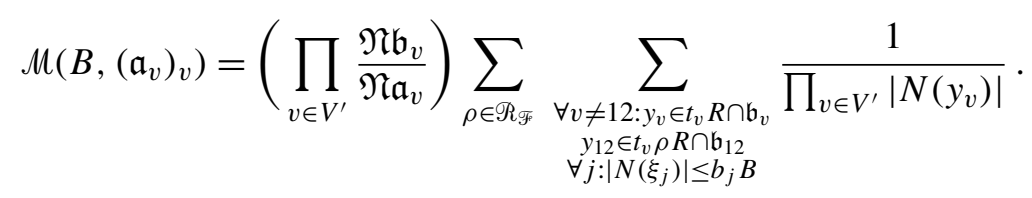


Again, the inner sum does not depend on the sets of representatives $t_{v} R, t_{v} \rho R$ for $K^{\times} / \mathscr{F}$. Thus,

$$
\mu\left(B,\left(\mathfrak{a}_{v}\right)_{v}\right)=3^{q}\left(\prod_{v \in V^{\prime}} \frac{\mathfrak{N \mathfrak { b } _ { v }}}{\mathfrak{N a}_{v}}\right) \sum_{\substack{y_{v} \in R \cap \mathfrak{b}_{v}, v \in V^{\prime} \\ \forall j:\left|N\left(\xi_{j}\right)\right| \leq b_{j} B}} \frac{1}{\prod_{v \in V^{\prime}}\left|N\left(y_{v}\right)\right|},
$$

where $R$ is any system of representatives for $K^{\times} / \mathscr{F}$. Let $\sigma: K \rightarrow \mathbb{R}^{r} \times \mathbb{C}^{s}$ be the standard embedding, and let $S_{F}^{1}(T)$ be defined as on page 1465 . We choose $R$ to be the set of all $y \in K^{\times}$with $\sigma(y) \in S_{F}^{1}(\infty)$. This is indeed a set of representatives for $K^{\times} / \mathscr{F}$ : For any $y \in K^{\times}, \zeta \in \mathscr{F}$, we have

$$
\left(d_{i} \log \left|\sigma_{i}(\zeta y)\right|\right)_{i=1}^{r+s}=\left(d_{i} \log \left|\sigma_{i}(y)\right|\right)_{i=1}^{r+s}+l(\zeta),
$$

and $F(\infty)$ is a system of representatives for the orbits of the additive action of $l(\mathscr{F})$ on $\mathbb{R}^{r+s}$. We will first consider the sum

$$
\mu_{1}\left(B,\left(\mathfrak{b}_{v}\right)_{v}\right):=\sum_{\substack{y_{v} \in R \cap \mathfrak{b}_{v}, v \in V^{\prime} \\ \forall j:\left|N\left(\xi_{j}\right)\right| \leq B}} \frac{1}{\prod_{v \in V^{\prime}}\left|N\left(y_{v}\right)\right|} .
$$

For any $z \in \mathbb{R}^{r} \times \mathbb{C}^{s}$, let $N(\boldsymbol{z}):=\left|z_{1}\right|^{d_{1}} \cdots\left|z_{r+s}\right|^{d_{r+s}}$. We define $M(B)$ as the set of all $\left(\boldsymbol{z}_{v}\right)_{v \in V^{\prime}} \in\left(\mathbb{R}^{r} \times \mathbb{C}^{s}\right)^{6}$ such that

for all $v \in V^{\prime}$, we have $z_{v} \in S_{F}^{1}(\infty)$ and $N\left(z_{v}\right) \geq 1$, and for all $j$, we have $N\left(z_{j k}\right) N\left(z_{j l}\right) N\left(z_{k j}\right)^{2} N\left(z_{l j}\right)^{2} \leq B$.

Then $M(B)$ is bounded for all $B$. Let $\Lambda$ be the lattice in $\left(\mathbb{R}^{r} \times \mathbb{C}^{s}\right)^{6}$ defined by

$$
\Lambda:=\prod_{v \in V^{\prime}} \sigma\left(\mathfrak{b}_{v}\right)
$$

By the componentwise extension of $\sigma$ to $K^{6}$, we obtain

$$
\mu_{1}\left(B,\left(\mathfrak{b}_{v}\right)_{v}\right)=\sum_{\left(z_{v}\right)_{v} \in \Lambda \cap M(B)} \frac{1}{\prod_{v \in V^{\prime}} N\left(z_{v}\right)} .
$$

We identify $\mathbb{C}$ with $\mathbb{R}^{2}$ and estimate this sum by an integral. Let

$$
I(B):=\left(\frac{2^{s}}{\sqrt{\left|\Delta_{K}\right|}}\right)^{6} \frac{1}{\prod_{v \in V^{\prime}} \mathfrak{N b}_{v}} \int_{M(B)} \prod_{v \in V^{\prime}} \frac{d \boldsymbol{z}_{v}}{N\left(\boldsymbol{z}_{v}\right)} .
$$

Lemma 5.4. We have

$$
\sum_{\left(z_{v}\right)_{v} \in \Lambda \cap M(B)} \frac{1}{\prod_{v \in V^{\prime}} N\left(z_{v}\right)}=I(B)+O\left((\log B)^{5}\right)
$$

for $B \geq e$. The implicit $O$-constant depends on $K$. 
Proof. This is a generalization of [Derenthal and Janda 2013, Lemma 5]. Let us fix some notation. For $v \in V^{\prime}$, let $F_{v}$ be a fundamental parallelotope for the lattice $\sigma\left(\mathfrak{b}_{v}\right) \subseteq \mathbb{R}^{r} \times \mathbb{C}^{s}=\mathbb{R}^{d}$, and let $R_{v}$ be the minimal $d$-dimensional interval containing $F_{v}$. We denote the side lengths of $R_{v}$ by $l_{v, 1}, \ldots, l_{v, d}$. For any $z=\left(z_{1}, \ldots, z_{d}\right) \in \mathbb{R}^{d}$ satisfying

$$
\left|z_{i}\right| \geq 1+l_{v, i} \text { for all } i \in\{1, \ldots, d\},
$$

let $R_{v}(z)$ be the (unique) translate of $R_{v}$ such that $z$ is the corner of $R_{v}(z)$ at utmost distance from the origin, and let $F_{v}(z)$ be the (unique) translate of $F_{v}$ contained in $R_{v}(z)$. Similarly, for any $z$ with

$$
\left|z_{i}\right| \geq 1 \text { for all } i \in\{1, \ldots, d\},
$$

let $R_{v}^{\prime}(z)$ be the (unique) translate of $R_{v}$ such that $z$ is the corner of $R_{v}^{\prime}(z)$ closest to the origin, and let $F_{v}^{\prime}(z)$ be the (unique) translate of $F_{v}$ contained in $R_{v}^{\prime}(z)$. Consistently with the above definition of $N(z)$ for $z \in \mathbb{R}^{r} \times \mathbb{C}^{s}$, we let

$$
N(z):=\left|z_{1} \cdots z_{r}\left(z_{r+1}^{2}+z_{r+2}^{2}\right) \cdots\left(z_{d-1}^{2}+z_{d}^{2}\right)\right| .
$$

Since $N(\boldsymbol{z}) \geq N(\boldsymbol{y})$ for all $\boldsymbol{y} \in F_{v}(\boldsymbol{z})$, we have

$$
\frac{1}{N(\boldsymbol{z})} \leq \frac{1}{\operatorname{Vol} F_{v}(\boldsymbol{z})} \int_{F_{v}(z)} \frac{d \boldsymbol{y}}{N(\boldsymbol{y})}=\frac{2^{s}}{\sqrt{\left|\Delta_{K}\right|} \mathfrak{N b}_{v}} \int_{F_{v}(z)} \frac{d \boldsymbol{y}}{N(\boldsymbol{y})}
$$

Similarly,

$$
\frac{1}{N(\boldsymbol{z})} \geq \frac{1}{\operatorname{Vol} F_{v}^{\prime}(\boldsymbol{z})} \int_{F_{v}^{\prime}(z)} \frac{d \boldsymbol{y}}{N(\boldsymbol{y})}=\frac{2^{s}}{\sqrt{\left|\Delta_{K}\right|} \mathfrak{N b}_{v}} \int_{F_{v}^{\prime}(z)} \frac{d \boldsymbol{y}}{N(\boldsymbol{y})} .
$$

Clearly, if $z \neq \mathbf{z}^{\prime} \in \sigma\left(\mathfrak{b}_{v}\right)$ with (5-12) then $F_{v}(z) \cap F_{v}\left(z^{\prime}\right)=\varnothing$. Let us first prove that

$$
\sum_{\left(z_{v}\right)_{v} \in \Lambda \cap M(B)} \frac{1}{\prod_{v \in V^{\prime}} N\left(z_{v}\right)} \leq I(B)+O\left((\log B)^{5}\right) .
$$

To this end, we define

$$
E(B):=\left\{\left(z_{v}\right)_{v} \in M(B) \mid \text { all } z_{v} \text { satisfy (5-12) and } F_{v}\left(z_{v}\right) \subseteq S_{F}^{1}(\infty)\right\},
$$

and $G(B):=M(B) \backslash E(B)$. Keep in mind that $E(B)$ and $G(B)$ depend on $\left(\mathfrak{b}_{v}\right)_{v \in V^{\prime}}$. For any $\left(z_{v}\right)_{v} \in \Lambda \cap E(B)$, we have $\prod_{v} F_{v}\left(z_{v}\right) \subseteq M(B)$. Therefore,

$$
\begin{aligned}
& \sum_{\left(z_{v}\right)_{v} \in \Lambda \cap E(B)} \frac{1}{\prod_{v \in V^{\prime}} N\left(z_{v}\right)} \leq \sum_{\left(z_{v}\right)_{v} \in \Lambda \cap E(B)} \prod_{v \in V^{\prime}} \frac{2^{s}}{\sqrt{\left|\Delta_{K}\right|} \mathfrak{N b}_{v}} \int_{F_{v}\left(z_{v}\right)} \frac{d \boldsymbol{y}}{N(\boldsymbol{y})} \\
& \leq\left(\frac{2^{s}}{\sqrt{\left|\Delta_{K}\right|}}\right)^{6} \frac{1}{\prod_{v \in V^{\prime}} \mathfrak{N b}_{v}} \sum_{\left(z_{v}\right)_{v} \in \Lambda \cap E(B)} \prod_{v \in V^{\prime}} \int_{F_{v}\left(z_{v}\right)} \frac{d \boldsymbol{z}_{v}}{N\left(z_{v}\right)} \leq I(B) .
\end{aligned}
$$


We need to prove that

$$
\sum_{\left(z_{v}\right)_{v} \in \Lambda \cap G(B)} \frac{1}{\prod_{v \in V^{\prime}} N\left(z_{v}\right)}=O\left((\log B)^{5}\right) .
$$

For every $\left(z_{v}\right)_{v} \in \Lambda \cap G(B)$, there is at least one $w \in V^{\prime}$ such that either

$$
z_{w} \text { does not satisfy (5-12) }
$$

or

$$
z_{w} \text { satisfies }(5-12) \text { and } F_{w}\left(z_{w}\right) \nsubseteq S_{F}^{1}(\infty) \text {. }
$$

Therefore, we have

$$
\begin{aligned}
& \sum_{\left(z_{v}\right)_{v} \in \Lambda \cap G(B)} \frac{1}{\prod_{v \in V^{\prime}} N\left(z_{v}\right)} \\
& \leq \sum_{w \in V^{\prime}} \sum_{\substack{\left(z_{v}\right)_{v} \in \Lambda \cap S_{F}^{1}(\infty)^{6} \\
(5-18) \text { or }(5-19)}} \frac{1}{\prod_{v \in V^{\prime}} N\left(z_{v}\right)} \\
& =\sum_{w \in V^{\prime}}\left(\prod_{v \neq w} \sum_{\substack{z \in \sigma\left(\mathfrak{b}_{v}\right) \cap S_{F}^{1}(\infty) \\
N(z) \leq B}} \frac{1}{N(\boldsymbol{z})}\right) \sum_{\substack{z \in \sigma\left(\mathfrak{b}_{w}\right) \cap S_{F}^{1}(\infty) \\
N(z) \leq B \\
(5-18)}} \frac{1}{N(\boldsymbol{z})} .
\end{aligned}
$$

Now

$$
\sum_{\substack{z \in \sigma\left(\mathfrak{b}_{v}\right) \cap S_{F}^{1}(\infty) \\ N(\boldsymbol{z}) \leq B}} \frac{1}{N(\boldsymbol{z})}=\omega_{K} \sum_{\substack{\{0\} \neq H \in P_{K} \\ H \subseteq \mathfrak{b}_{v} \\ \mathfrak{N} H \leq B}} \frac{1}{\mathfrak{N} H} \leq \sum_{\substack{\{0\} \neq H \unlhd \mathcal{O}_{K} \\ \mathfrak{N} H \leq B}} \frac{1}{\mathfrak{N} H} \ll \log B,
$$

by Lemma 5.3. Moreover, we write

$$
\sum_{\substack{z \in \sigma\left(\mathfrak{b}_{w}\right) \cap S_{F}^{1}(\infty) \\ N(z) \leq B \\(5-18) \text { or }(5-19) \text { for } z}} \frac{1}{N(z)}=\sum_{n=1}^{B} a_{n} \cdot \frac{1}{n},
$$

with $a_{n}:=\mid\left\{z \in \sigma\left(\mathfrak{b}_{w}\right) \cap S_{F}^{1}(\infty) \mid N(\boldsymbol{z})=n,(5-18)\right.$ or (5-19) holds for $\left.z\right\} \mid$. We will apply the Abel sum formula, so we need to understand

$$
A(T):=\sum_{n \leq T} a_{n}=\mid\left\{z \in \sigma\left(\mathfrak{b}_{w}\right) \cap S_{F}^{1}\left(T^{1 / d}\right) \mid(5-18) \text { or (5-19) holds for } z\right\} \mid .
$$

Let

$$
H:=\left\{z \in \mathbb{R}^{d} \mid z_{1} \cdots z_{d}=0\right\}
$$


and let $D_{w}$ be the $d$-dimensional interval

$$
D_{w}:=\left[-\left(l_{w, 1}+1\right), l_{w, 1}+1\right] \times \cdots \times\left[-\left(l_{w, d}+1\right), l_{w, d}+1\right] \subseteq \mathbb{R}^{d} .
$$

Then any $z$ counted by $A(T)$ satisfies $\left(z+D_{w}\right) \cap H \neq \varnothing$ (if (5-18) holds) or $z+D_{w} \nsubseteq S_{F}^{1}\left(T^{1 / d}\right)$ (if (5-19) holds). Therefore, any such $z$ is contained in $A_{1}(T) \cup A_{2}(T)$, where

$$
\begin{aligned}
A_{1}(T): & =\left\{z \in \sigma\left(\mathfrak{b}_{w}\right) \mid\left(z+D_{w}\right) \cap \partial S_{F}^{1}\left(T^{1 / d}\right) \neq \varnothing\right\} \\
& \supseteq\left\{z \in \sigma\left(\mathfrak{b}_{w}\right) \cap S_{F}^{1}\left(T^{1 / d}\right) \mid\left(z+D_{w}\right) \nsubseteq S_{F}^{1}\left(T^{1 / d}\right)\right\}, \\
A_{2}(T): & =\left\{z \in \sigma\left(\mathfrak{b}_{w}\right) \mid\left(z+D_{w}\right) \cap\left(S_{F}^{1}\left(T^{1 / d}\right) \cap H\right) \neq \varnothing\right\} \\
& \supseteq\left\{z \in \sigma\left(\mathfrak{b}_{w}\right) \cap S_{F}^{1}\left(T^{1 / d}\right) \mid\left(z+D_{w}\right) \subseteq S_{F}^{1}\left(T^{1 / d}\right),\left(z_{w}+D_{w}\right) \cap H \neq \varnothing\right\} .
\end{aligned}
$$

Now $\partial S_{F}^{1}\left(T^{1 / d}\right)=T^{1 / d} \partial S_{F}^{1}(1) \in \operatorname{Lip}\left(d, M_{1}, T^{1 / d} L_{1}\right)$. We recall that $\mathfrak{b}_{v} \in \mathscr{C}$, so Lemma 4.1(i) implies that

$$
\left|A_{1}(T)\right| \ll M_{1}\left(L_{1} T^{1 / d}+1\right)^{d-1} \ll T^{(d-1) / d} \quad \text { for all } T \geq 1 .
$$

Moreover, $S_{F}^{1}\left(T^{1 / d}\right) \cap H=T^{1 / d}\left(S_{F}^{1}(1) \cap H\right)$, and clearly $S_{F}^{1}(1) \cap H \in \operatorname{Lip}\left(d, \tilde{M}_{1}, \tilde{L}_{1}\right)$ for some $\tilde{M}_{1}$ and $\tilde{L}_{1}$. By Lemma 4.1(i),

$$
\left|A_{2}(T)\right| \ll \tilde{M}_{1}\left(\tilde{L}_{1} T^{1 / d}+1\right)^{d-1} \ll T^{(d-1) / d} \quad \text { for all } T \geq 1 .
$$

Therefore, $A(T) \ll T^{(d-1) / d}$ for $T \geq 1$. The Abel sum formula yields

$$
\sum_{n=1}^{B} a_{n} \cdot \frac{1}{n}=A(B) / B+\int_{t=1}^{B} A(t) / t^{2} d t \ll B^{-1 / d}+\int_{t=1}^{B} t^{-(1+1 / d)} d t \ll 1 .
$$

With (5-20), (5-21), (5-22), we see that (5-17) holds, which finishes the proof of (5-16). Let us prove the other inequality, that is

$$
I(B) \leq \sum_{\left(z_{v}\right)_{v} \in \Lambda \cap M(B)} \frac{1}{\prod_{v \in V^{\prime}} N\left(z_{v}\right)}+O\left((\log B)^{5}\right) .
$$

For every $v \in V^{\prime}$ and every $z \in \mathbb{R}^{d}$ satisfying (5-12), there is a unique $\lambda_{v}(\boldsymbol{z}) \in \sigma\left(\mathfrak{b}_{v}\right)$ with (5-13) such that $z \in F_{v}^{\prime}\left(\lambda_{v}(z)\right)$. In a similar way as above, we define

$$
E^{\prime}(B):=\left\{\left(z_{v}\right)_{v} \in M(B) \mid \text { all } z_{v} \text { satisfy (5-12) and } \lambda_{v}\left(z_{v}\right) \in S_{F}^{1}(\infty)\right\},
$$

and $G^{\prime}(B):=M(B) \backslash E^{\prime}(B)$. Both $E^{\prime}(B)$ and $G^{\prime}(B)$ are clearly measurable. For any $\left(z_{v}\right)_{v}$ in $E^{\prime}(B)$, the point $\left(\lambda_{v}\left(z_{v}\right)\right)_{v}$ is the unique element of $\Lambda \cap M(B)$ with 
$z_{v} \in F_{v}^{\prime}\left(\lambda_{v}\left(z_{v}\right)\right)$ for all $v \in V^{\prime}$. With this and (5-15), we obtain

$$
\begin{aligned}
\frac{2^{6 s}}{\left(\sqrt{\left|\Delta_{K}\right|}\right)^{6} \prod_{v \in V^{\prime}} \mathfrak{N b}_{v}} \int_{E^{\prime}(B)} \prod_{v \in V^{\prime}} \frac{d z_{v}}{N\left(z_{v}\right)} & \leq \sum_{\substack{\left(\lambda_{v}\right)_{v} \in \\
\Lambda \cap M(B)}} \prod_{v \in V^{\prime}} \frac{2^{s}}{\sqrt{\left|\Delta_{K}\right|} \mathfrak{N b}_{v}} \int_{F_{v}^{\prime}\left(\lambda_{v}\right)} \frac{d z}{N(z)} \\
& \leq \sum_{\left(\lambda_{v}\right)_{v} \in \Lambda \cap M(B)} \frac{1}{\prod_{v \in V^{\prime}} N\left(\lambda_{v}\right)}
\end{aligned}
$$

We need to prove that

$$
\left(\frac{2^{s}}{\sqrt{\left|\Delta_{K}\right|}}\right)^{6} \frac{1}{\prod_{v \in V^{\prime}} \mathfrak{N b}_{v}} \int_{G^{\prime}(B)} \prod_{v \in V^{\prime}} \frac{d z_{v}}{N\left(z_{v}\right)}=O\left((\log B)^{5}\right)
$$

For every $\left(z_{v}\right)_{v} \in G^{\prime}(B)$, there is some $w \in V^{\prime}$ such that either

$$
z_{w} \text { does not satisfy }(5-12)
$$

or

$$
\boldsymbol{z}_{w} \text { satisfies }(5-12) \text { and } \lambda_{w}\left(z_{w}\right) \notin S_{F}^{1}(\infty) \text {. }
$$

Similarly to (5-20), we obtain

$$
\int_{G^{\prime}(B)} \prod_{v \in V^{\prime}} \frac{d z_{v}}{N\left(z_{v}\right)} \leq \sum_{w \in V^{\prime}}\left(\prod_{v \neq w} \int_{\substack{z \in S_{F}^{1}(\infty) \\ 1 \leq N(z) \leq B}} \frac{d z}{N(z)}\right) \int_{\substack{z \in S_{F}^{1}(\infty) \\ 1 \leq N(z) \leq B \\(5-28) \text { or }(5-29) \text { for } z}} \frac{d z}{N(z)}
$$

We denote the Lebesgue measure on $\mathbb{R}, \mathbb{R}^{d}$ by $m_{1}, m_{d}$. The restriction of $N$ to $S_{F}^{1}(\infty)$ defines a measurable function $N_{1}: S_{F}^{1}(\infty) \rightarrow \mathbb{R}$. Since

$$
\left(m_{d} \circ N_{1}^{-1}\right)((a, b])=\operatorname{Vol} S_{F}^{1}\left(b^{1 / d}\right)-\operatorname{Vol} S_{F}^{1}\left(a^{1 / d}\right)=(b-a) \operatorname{Vol} S_{F}^{1}(1)
$$

for all $0<a \leq b \in \mathbb{R}$, we obtain $m_{d} \circ N_{1}^{-1}=\operatorname{Vol} S_{F}^{1}(1) m_{1}$ on $\mathbb{R}^{>0}$. Therefore,

$$
\int_{\substack{z \in S_{F}^{1}(\infty) \\ 1 \leq N(z) \leq B}} \frac{d z}{N(z)}=\int_{N_{1}^{-1}([1, B])} \frac{d m_{d}}{N_{1}(z)}=\int_{[1, B]} \frac{1}{t} d\left(m_{d} \circ N_{1}^{-1}\right)=\operatorname{Vol} S_{F}^{1}(1) \log B .
$$

Let $A(T):=\left\{z \in S_{F}^{1}(\infty) \mid 1 \leq N(z) \leq T,(5-28)\right.$ or (5-29) holds for $\left.z\right\}$. Then $A(T)$ is measurable for all $T$ and the restriction of $N$ to $A(B)$ defines a measurable function $N_{2}: A(B) \rightarrow[1, B]$. For any $E \subseteq[1, B]$ with $m_{1}(E)=0$, we have $N_{2}^{-1}(E) \subseteq N_{1}^{-1}(E)$ and $\left(m_{d} \circ N_{1}^{-1}\right)(E)=0$. Thus, $m_{d} \circ N_{2}^{-1}$ is absolutely continuous. With the distribution function $F(T):=\left(m_{d} \circ N_{2}^{-1}\right)([1, T])$, we obtain

$$
\int_{A(B)} \frac{d z}{N(z)}=\int_{N_{2}^{-1}([1, B])} \frac{d m_{d}}{N_{2}(z)}=\int_{[1, B]} \frac{1}{t} d\left(m_{d} \circ N_{2}^{-1}\right)=\int_{1}^{B} \frac{1}{t} d F(t) .
$$


Integration by parts for the Stieltjes integral on the right-hand side suggests that we need to find a suitable bound for $F(T)$. Clearly,

$$
F(T)=\operatorname{Vol}\left(N_{2}^{-1}([1, T])\right)=\operatorname{Vol} A(T) .
$$

With $H, D_{w}$ as in (5-23), (5-24), let

$$
\begin{aligned}
& A_{1}(T):=\left\{z \in \mathbb{R}^{d} \mid\left(z+D_{w}\right) \cap \partial S_{F}^{1}\left(T^{1 / d}\right) \neq \varnothing\right\}, \\
& A_{2}(T):=\left\{z \in \mathbb{R}^{d} \mid\left(z+D_{w}\right) \cap\left(\overline{S_{F}^{1}\left(T^{1 / d}\right)} \cap H\right) \neq \varnothing\right\} .
\end{aligned}
$$

A similar argument to before shows that $A(T) \subseteq A_{1}(T) \cup A_{2}(T)$. We already know that $\partial S_{F}^{1}\left(T^{1 / d}\right) \in \operatorname{Lip}\left(d, M_{1}, T^{1 / d} L_{1}\right)$ and $S_{F}^{1}\left(T^{1 / d}\right) \cap H \in \operatorname{Lip}\left(n, \tilde{M}_{1}, T^{1 / d} \tilde{L}_{1}\right)$. The same holds of course for the closure. By Lemma 4.1(ii) we obtain

$$
\operatorname{Vol} A_{1}(T) \ll T^{(d-1) / d}, \quad \operatorname{Vol} A_{2}(T) \ll T^{(d-1) / d} \text { for } T \geq 1,
$$

and thus $F(T) \ll T^{(d-1) / d}$ for $T \geq 1$. Integration by parts gives

$$
\int_{1}^{B} \frac{1}{t} d F(t)=F(B) / B-F(1)-\int_{1}^{B} F d \frac{1}{t} \ll B^{-1 / d}+\int_{1}^{B} t^{-(1+1 / d)} d t \ll 1 .
$$

With (5-30), (5-31) and (5-32), we obtain (5-27). Together with (5-26) this gives (5-25).

Lemma 5.5. We have

$$
I(B)=\frac{1}{4 \cdot 6 !}\left(\frac{2^{r}(2 \pi)^{s} R_{K}}{\sqrt{\left|\Delta_{K}\right|}}\right)^{6} \frac{1}{\prod_{v \in V^{\prime}} \mathfrak{N b}_{v}}(\log B)^{6} .
$$

Proof. Let $m_{n}$ denote the Lebesgue measure on $\mathbb{R}^{n}$. We define the measurable function $f:\left(S_{F}^{1}(\infty)\right)^{6} \rightarrow \mathbb{R}^{6}$ by $f\left(\left(z_{v}\right)_{v \in V^{\prime}}\right)=\left(N\left(z_{v}\right)\right)_{v \in V^{\prime}}$. For any cell $E:=\prod_{v \in V^{\prime}}\left(a_{v}, b_{v}\right]$, with $0<a_{v} \leq b_{v}$, we have

$$
\left(m_{6 d} \circ f^{-1}\right)(E)=\prod_{v \in V^{\prime}}\left(\operatorname{Vol} S_{F}^{1}\left(b_{v}^{1 / d}\right)-\operatorname{Vol} S_{F}^{1}\left(a_{v}^{1 / d}\right)\right)=\left(\operatorname{Vol} S_{F}^{1}(1)\right)^{6} m_{6}(E) .
$$

Thus, $m_{6 d} \circ f^{-1}=\left(\operatorname{Vol} S_{F}^{1}(1)\right)^{6} m_{6}$ on $\left(\mathbb{R}^{\geq 0}\right)^{6}$. Let

$$
M_{\mathbb{Q}}(B):=\left\{\left(t_{v}\right)_{v \in V^{\prime}} \in \mathbb{R}^{6} \mid t_{v} \geq 1 \text { for all } v \text { and } t_{j k} t_{j l} t_{k j}^{2} t_{l j}^{2} \leq B \text { for all } j\right\} .
$$

Then

$$
\begin{aligned}
\int_{M(B)} \prod_{v \in V^{\prime}} \frac{d z_{v}}{N\left(z_{v}\right)} & =\int_{f^{-1}\left(M_{\mathbb{Q}}(B)\right)} \prod_{v \in V^{\prime}} \frac{1}{f(z)_{v}} d m_{6 d}=\int_{M_{\mathbb{Q}}(B)} \prod_{v \in V^{\prime}} \frac{1}{t_{v}} d\left(m_{6 d} \circ f^{-1}\right) \\
& =\left(\operatorname{Vol} S_{F}^{1}(1)\right)^{6} \int_{M_{\mathbb{Q}}(B)} \prod_{v \in V^{\prime}} \frac{1}{t_{v}} d m_{6}=\frac{\left(\operatorname{Vol} S_{F}^{1}(1)\right)^{6}}{4 \cdot 6 !}(\log B)^{6} .
\end{aligned}
$$

The last integral is computed at the end of [Heath-Brown and Moroz 1999]. 
We define

$$
C_{0}(K):=\frac{1}{4 \cdot 6 !}\left(\frac{2^{r}(2 \pi)^{s} R_{K}}{\sqrt{\left|\Delta_{K}\right|}}\right)^{6} \text { and } C(K):=3^{q} C_{0}(K) .
$$

Then (5-11) and the previous two lemmata imply that

$$
\mu_{1}\left(B,\left(\mathfrak{b}_{v}\right)_{v}\right)=\frac{C_{0}(K)}{\prod_{v \in V^{\prime}} \mathfrak{N b _ { v }}}(\log B)^{6}+O(\log B)^{5} .
$$

Keep in mind that $\mathfrak{b}_{v} \in \mathscr{C}$ for all $v \in V^{\prime}$. With (5-9), (5-10), we obtain

$$
\mathcal{M}\left(B,\left(\mathfrak{a}_{v}\right)_{v}\right) \leq \frac{C(K)}{\prod_{v \in V^{\prime}} \mathfrak{N a}_{v}}(\log B)^{6}+O\left(\frac{1}{\prod_{v \in V^{\prime}} \mathfrak{N a}_{v}}(\log B)^{5}\right) .
$$

Let $R:=\max _{j}\left\{\mathfrak{N a}_{j}\right\}^{1 / d} \prod_{v \in V^{\prime}} \mathfrak{N a}_{v}^{2 /(3 d)}$. Then $R \geq c_{4}>0$ for some constant $c_{4}$ depending only on $K$. This implies in particular that $\log R \ll R$. Moreover, we have $1 /\left(c_{5} R^{3 d}\right) \leq b_{j}$ for some constant $c_{5} \geq 1$ depending only on $K$. Therefore,

$$
\mu\left(B,\left(\mathfrak{a}_{v}\right)_{v}\right) \geq 3^{q}\left(\prod_{v \in V^{\prime}} \frac{\mathfrak{N b}_{v}}{\mathfrak{N a}_{v}}\right) M_{1}\left(B /\left(c_{5} R^{3 d}\right),\left(\mathfrak{b}_{v}\right)_{v}\right) .
$$

Whenever $B \geq e c_{5} R^{3 d}$, we obtain

$$
\begin{aligned}
\mathcal{M}\left(B,\left(\mathfrak{a}_{v}\right)_{v}\right) & \geq \frac{C(K)}{\prod_{v \in V^{\prime}} \mathfrak{N a _ { v }}} \log \left(B /\left(c_{5} R^{3 d}\right)\right)^{6}+O\left(\frac{1}{\prod_{v \in V^{\prime}} \mathfrak{N a}_{v}} \log \left(B /\left(c_{5} R^{3 d}\right)\right)^{5}\right) \\
& =\frac{C(K)}{\prod_{v \in V^{\prime}} \mathfrak{N a}_{v}}(\log B)^{6}+O\left(\frac{R}{\prod_{v \in V^{\prime}} \mathfrak{N a}_{v}}(\log B)^{5}\right) .
\end{aligned}
$$

This result holds as well if $e \leq B<e c_{5} R^{3 d}$, since then the error term dominates the main term. Therefore,

$$
\mathcal{M}\left(B,\left(\mathfrak{a}_{v}\right)_{v}\right)=\frac{C(K)}{\prod_{v \in V^{\prime}} \mathfrak{N a a _ { v }}}(\log B)^{6}+O\left(\frac{R}{\prod_{v \in V^{\prime}} \mathfrak{N a} \mathfrak{a}_{v}}(\log B)^{5}\right),
$$

and Lemma 3.1 follows from (5-8).

\section{References}

[Baier and Browning 2013] S. Baier and T. D. Browning, "Inhomogeneous cubic congruences and rational points on Del Pezzo surfaces", J. Reine Angew. Math. 680 (2013), 69-151.

[Batyrev and Tschinkel 1998a] V. V. Batyrev and Y. Tschinkel, "Manin's conjecture for toric varieties", J. Algebraic Geom. 7:1 (1998), 15-53. MR 2000c:11107 Zbl 0946.14009

[Batyrev and Tschinkel 1998b] V. V. Batyrev and Y. Tschinkel, "Tamagawa numbers of polarized algebraic varieties", pp. 299-340 in Nombre et répartition de points de hauteur bornée (Paris, 1996), Astérisque 251, Société Mathématique de France, Paris, 1998. MR 2000d:11090 Zbl 0926.11045 
[de la Bretèche 1998] R. de la Bretèche, "Sur le nombre de points de hauteur bornée d'une certaine surface cubique singulière", pp. 51-77 in Nombre et répartition de points de hauteur bornée (Paris, 1996), Astérisque 251, Société Mathématique de France, Paris, 1998. MR 2000b:11074 Zbl 0969.14014

[de la Bretèche 2002] R. de la Bretèche, "Nombre de points de hauteur bornée sur les surfaces de del Pezzo de degré 5”, Duke Math. J. 113:3 (2002), 421-464. MR 2003m:14033 Zbl 1054.14025

[de la Bretèche and Browning 2011] R. de la Bretèche and T. D. Browning, "Manin's conjecture for quartic del Pezzo surfaces with a conic fibration", Duke Math. J. 160:1 (2011), 1-69. MR 2012k:11038 Zbl 1245.11044

[de la Bretèche and Fouvry 2004] R. de la Bretèche and É. Fouvry, "L'éclaté du plan projectif en quatre points dont deux conjugués”, J. Reine Angew. Math. 576 (2004), 63-122. MR 2005f:11131 Zbl 1065.11080

[de la Bretèche and Swinnerton-Dyer 2007] R. de la Bretèche and P. Swinnerton-Dyer, "Fonction zêta des hauteurs associée à une certaine surface cubique”, Bull. Soc. Math. France 135:1 (2007), 65-92. MR 2009f:14041 Zbl 1207.11068

[de la Bretèche et al. 2007] R. de la Bretèche, T. D. Browning, and U. Derenthal, “On Manin's conjecture for a certain singular cubic surface", Ann. Sci. École Norm. Sup. (4) 40:1 (2007), 1-50. MR 2008e:11038 Zbl 1125.14008

[de la Bretèche et al. 2012] R. de la Bretèche, T. Browning, and E. Peyre, "On Manin's conjecture for a family of Châtelet surfaces”, Ann. of Math. (2) 175:1 (2012), 297-343. MR 2874644 Zbl 1237.11018

[Browning and Derenthal 2009] T. D. Browning and U. Derenthal, "Manin's conjecture for a cubic surface with $D_{5}$ singularity", Int. Math. Res. Not. 2009:14 (2009), 2620-2647. MR 2011a:14041 Zbl 1173.14017

[Chambert-Loir and Tschinkel 2002] A. Chambert-Loir and Y. Tschinkel, "On the distribution of points of bounded height on equivariant compactifications of vector groups", Invent. Math. 148:2 (2002), 421-452. MR 2003d:11094 Zbl 1067.11036

[Christensen and Gubler 2008] C. Christensen and W. Gubler, "Der relative Satz von Schanuel", Manuscripta Math. 126:4 (2008), 505-525. MR 2009e:11124 Zbl 1155.11034

[Colliot-Thélène and Sansuc 1980] J.-L. Colliot-Thélène and J.-J. Sansuc, "La descente sur les variétés rationnelles", pp. 223-237 in Journées de Géometrie Algébrique d'Angers, Juillet 1979/Algebraic Geometry, Angers, 1979, edited by A. Beauville, Sijthoff \& Noordhoff, Alphen aan den Rijn, 1980. MR 82d:14016 Zbl 0451.14018

[Colliot-Thélène and Sansuc 1987] J.-L. Colliot-Thélène and J.-J. Sansuc, "La descente sur les variétés rationnelles, II”, Duke Math. J. 54:2 (1987), 375-492. MR 89f:11082 Zbl 0659.14028

[Derenthal and Janda 2013] U. Derenthal and F. Janda, "Gaussian rational points on a singular cubic surface", pp. 210-230 in Torsors, étale homotopy and applications to rational points (Edinburgh, 2011), London Math. Soc. Lecture Note Series 405, Cambridge Univ. Press, 2013.

[Fouvry 1998] É. Fouvry, "Sur la hauteur des points d'une certaine surface cubique singulière", pp. 31-49 in Nombre et répartition de points de hauteur bornée (Paris, 1996), Astérisque 251, Société Mathématique de France, Paris, 1998. MR 2000b:11075 Zbl 0930.11044

[Franke et al. 1989] J. Franke, Y. I. Manin, and Y. Tschinkel, "Rational points of bounded height on Fano varieties”, Invent. Math. 95:2 (1989), 421-435. MR 89m:11060 Zbl 0674.14012

[Heath-Brown and Moroz 1999] D. R. Heath-Brown and B. Z. Moroz, "The density of rational points on the cubic surface $X_{0}^{3}=X_{1} X_{2} X_{3}$ ", Math. Proc. Cambridge Philos. Soc. 125:3 (1999), 385-395. MR 2000f:11080 Zbl 0938.11016 
[Lang 1994] S. Lang, Algebraic number theory, 2nd ed., Graduate Texts in Mathematics 110, Springer, New York, 1994. MR 95f:11085 Zbl 0811.11001

[Le Boudec 2012] P. Le Boudec, "Manin's conjecture for a cubic surface with $2 A_{2}+A_{1}$ singularity type", Math. Proc. Cambridge Philos. Soc. 153:3 (2012), 419-455.

[Masser and Vaaler 2007] D. Masser and J. D. Vaaler, "Counting algebraic numbers with large height. II”, Trans. Amer. Math. Soc. 359:1 (2007), 427-445. MR 2008m:11208 Zbl 1215.11100

[Peyre 1995] E. Peyre, "Hauteurs et mesures de Tamagawa sur les variétés de Fano", Duke Math. J. 79:1 (1995), 101-218. MR 96h:11062 Zbl 0901.14025

[Salberger 1998] P. Salberger, "Tamagawa measures on universal torsors and points of bounded height on Fano varieties", pp. 91-258 in Nombre et répartition de points de hauteur bornée (Paris, 1996), Astérisque 251, Société Mathématique de France, Paris, 1998. MR 2000d:11091 Zbl 0959.14007

[Schanuel 1979] S. H. Schanuel, "Heights in number fields", Bull. Soc. Math. France 107:4 (1979), 433-449. MR 81c:12025 Zbl 0428.12009

[Widmer 2010] M. Widmer, "Counting primitive points of bounded height", Trans. Amer. Math. Soc. 362:9 (2010), 4793-4829. MR 2011i:11099 Zbl 05791771

Communicated by Jean-Louis Colliot-Thélène

Received 2012-04-10 Revised 2012-07-30 Accepted 2012-09-07

frei@math.Imu.de

Mathematisches Institut, LMU München, Theresienstr. 39, D-80333 München, Germany

http://www.mathematik.uni-muenchen.de/ frei 


\section{Algebra \& Number Theory}

msp.org/ant

\section{EDITORS}

MANAGING EDITOR

Bjorn Poonen

Massachusetts Institute of Technology

Cambridge, USA

\author{
EDITORIAL BOARD CHAIR \\ David Eisenbud \\ University of California \\ Berkeley, USA
}

\section{BOARD OF EDITORS}

Georgia Benkart

Dave Benson

Richard E. Borcherds

John H. Coates

J-L. Colliot-Thélène

Brian D. Conrad

Hélène Esnault

Hubert Flenner

Edward Frenkel

Andrew Granville

Joseph Gubeladze

Ehud Hrushovski

Craig Huneke

Mikhail Kapranov

Yujiro Kawamata

János Kollár

Yuri Manin

Barry Mazur

Philippe Michel
University of Wisconsin, Madison, USA

University of Aberdeen, Scotland

University of California, Berkeley, USA

University of Cambridge, UK

CNRS, Université Paris-Sud, France

University of Michigan, USA

Freie Universität Berlin, Germany

Ruhr-Universität, Germany

University of California, Berkeley, USA

Université de Montréal, Canada

San Francisco State University, USA

Hebrew University, Israel

University of Virginia, USA

Yale University, USA

University of Tokyo, Japan

Princeton University, USA

Northwestern University, USA

Harvard University, USA

École Polytechnique Fédérale de Lausanne
Susan Montgomery

Shigefumi Mori

Raman Parimala

Jonathan Pila

Victor Reiner

Karl Rubin

Peter Sarnak

Joseph H. Silverman

Michael Singer

Vasudevan Srinivas

J. Toby Stafford

Bernd Sturmfels

Richard Taylor

Ravi Vakil

Michel van den Bergh

Marie-France Vignéras

Kei-Ichi Watanabe

Efim Zelmanov
University of Southern California, USA

RIMS, Kyoto University, Japan

Emory University, USA

University of Oxford, UK

University of Minnesota, USA

University of California, Irvine, USA

Princeton University, USA

Brown University, USA

North Carolina State University, USA

Tata Inst. of Fund. Research, India

University of Michigan, USA

University of California, Berkeley, USA

Harvard University, USA

Stanford University, USA

Hasselt University, Belgium

Université Paris VII, France

Nihon University, Japan

University of California, San Diego, USA

\section{PRODUCTION}

production@msp.org

Silvio Levy, Scientific Editor

See inside back cover or msp.org/ant for submission instructions.

The subscription price for 2013 is US \$200/year for the electronic version, and \$350/year ( $\$ 40$, if shipping outside the US) for print and electronic. Subscriptions, requests for back issues and changes of subscribers address should be sent to MSP.

Algebra \& Number Theory (ISSN 1944-7833 electronic, 1937-0652 printed) at Mathematical Sciences Publishers, 798 Evans Hall \#3840, c/o University of California, Berkeley, CA 94720-3840 is published continuously online. Periodical rate postage paid at Berkeley, CA 94704, and additional mailing offices.

ANT peer review and production are managed by EditFLOW ${ }^{\circledR}$ from Mathematical Sciences Publishers.

\section{PUBLISHED BY}

- mathematical sciences publishers

nonprofit scientific publishing

http://msp.org/

(C) 2013 Mathematical Sciences Publishers 


\section{Algebra \& Number Theory}

Volume $7 \quad$ No. $6 \quad 2013$

On the discrete logarithm problem in elliptic curves II

Claus Diem

Identifying Frobenius elements in Galois groups

1325

TIM DOKCHITSER and VLADIMIR DOKCHITSER

Weak approximation for cubic hypersurfaces of large dimension

1353

MIKE SWARBRICK JONES

The Picard crossed module of a braided tensor category

1365

ALEXEI DAVYDOV and DMITRI NIKSHYCH

A Gross-Zagier formula for quaternion algebras over totally real fields

1405

EYAL Z. GOREN and KRISTIN E. LAUTER

Counting rational points over number fields on a singular cubic surface

CHRISTOPHER FREI

On the ample cone of a rational surface with an anticanonical cycle

ROBERT FRIEDMAN

Commuting involutions of Lie algebras, commuting varieties, and simple Jordan algebras

DMITRI I. PANYUSHEV 